\title{
THE HEASARC Swift GAMMA-RAY BURST ARCHIVE: THE PIPELINE AND THE CATALOG
}

\author{
D. Donato ${ }^{1,2}$, L. Angelini ${ }^{3}$, C. A. Padgett ${ }^{4}$, T. Reichard ${ }^{5}$, N. Gehrels ${ }^{3}$, F. E. Marshall ${ }^{3}$, And T. SAKAmoto $^{1,6}$ \\ ${ }^{1}$ Center for Research and Exploration in Space Science and Technology (CRESST) and NASA Goddard Space Flight Center, \\ Greenbelt, MD 20771, USA; davide.donato-1@nasa.gov \\ 2 Department of Physics and Department of Astronomy, University of Maryland, College Park, MD 20742, USA \\ ${ }^{3}$ NASA Goddard Space Flight Center, Greenbelt, MD 20771, USA \\ ${ }^{4}$ RS Information Systems, Inc., McLean, VA 22102, USA \\ ${ }^{5}$ ADNET Systems, Inc., Rockville, MD 20852, USA \\ ${ }^{6}$ Joint Center for Astrophysics, University of Maryland, Baltimore County, 1000 Hilltop Circle, Baltimore, MD 21250, USA \\ Received 2012 June 22; accepted 2012 August 9; published 2012 October 11
}

\begin{abstract}
Since its launch in late 2004, the Swift satellite triggered or observed an average of one gamma-ray burst (GRB) every 3 days, for a total of 771 GRBs by 2012 January. Here, we report the development of a pipeline that semiautomatically performs the data-reduction and data-analysis processes for the three instruments on board Swift (BAT, XRT, UVOT). The pipeline is written in Perl, and it uses only HEAsoft tools and can be used to perform the analysis of a majority of the point-like objects (e.g., GRBs, active galactic nuclei, pulsars) observed by Swift. We run the pipeline on the GRBs, and we present a database containing the screened data, the output products, and the results of our ongoing analysis. Furthermore, we created a catalog summarizing some GRB information, collected either by running the pipeline or from the literature. The Perl script, the database, and the catalog are available for downloading and querying at the HEASARC Web site.
\end{abstract}

Key words: catalogs - methods: data analysis - X-rays: bursts

Online-only material: color figures

\section{INTRODUCTION}

Since the first gamma-ray burst (GRB) was observed by the Vela satellite (Klebesadel et al. 1973), the number of flashes of high-energy radiation that has been detected has increased dramatically, especially since the 1991 launch of the Compton Gamma Ray Observatory and its $\gamma$-ray instrument, the Burst and Transient Source Experiment instrument (Meegan et al. 1992). Discoveries made by the BeppoSAX satellite starting in 1997 showed that GRB afterglows are strong sources across a broad wavelength band (Sahu et al. 1997; Wijers et al. 1997).

In 2004 November, NASA launched the Swift satellite (Gehrels et al. 2004), which has the ability to detect a GRB with its $\gamma$-ray instrument (the Burst Alert Telescope, BAT; Barthelmy et al. 2005), to rapidly slew to the $\gamma$-ray position, and to observe the GRB afterglow with the X-Ray Telescope (XRT; Burrows et al. 2005) and the Ultraviolet/Optical Telescope (UVOT; Roming et al. 2005). Through the end of 2012 January, Swift observed 771 GRBs, an average of three GRBs detected every 10 days. The analysis of the data is performed by the Swift instrument teams, with each team focusing on their instrument. The results of these analyses are reported in the Gamma-ray Coordinates Network (GCN) circulars, a very effective way of informing the GRB research community of the initial properties of GRBs. Although the circulars have an important role, they also show some limitations. (1) The Swift data analysis usually refers to an earlier part of the GRB observation (typically coinciding with the first few orbits), and only in a limited number of cases are the results from followup observations released. Furthermore, analysis methods have evolved and this may make some circulars partially incomplete, the results not uniform and, consequently, more difficult to compare. (2) The analysis in the GCN circulars is done with the software and calibration available at the time of the burst, and may not be updated when newer versions are released. These limitations suggested to us that an archive containing the re- sults of the data analysis of every observed GRB was desierable. The following Swift instrument teams have processed data from their instruments: the NASA Goddard (GSFC) BAT Team; the UK Swift Science Data Centre at the University of Leicester for the XRT; and the UVOT team at Penn State University, NASA/GSFC, and the Mullard Space Science Laboratory. Each group focused on one specific instrument and a summary of their analysis is collected at the Swift Burst Ground-Analysis Web site (http://gcn.gsfc.nasa.gov/swift_gnd_ana.html), while a more complete database is available at the University of Leicester Web site (http://www.swift.ac.uk/xrt_live_cat/) for the XRT and at the "First Swift UVOT GRB Afterglow Catalog" Web site at NASA/GSFC (http://swift.gsfc.nasa.gov/docs/swift/results/ uvot_grbcat/) for the UVOT. The methods adopted by the BAT, XRT, and UVOT teams, as well as some of their results, have been extensively described (Sakamoto et al. 2008, 2011 for the BAT; Evans et al. 2007, 2009, 2010, and Goad et al. 2007 for the XRT; Poole et al. 2008 and Roming et al. 2005, 2009 for the UVOT).

In this paper, we present a pipeline that semi-automatically performs the data reduction and analysis of all the Swift instruments. We run the pipeline to analyze all the GRBs observed by Swift during its first 7 years of operation, and we describe the key products available for downloading. We also present our Swift GRB Catalog, whose purpose is to collect some basic information related to each GRB, retrieved from the analysis performed by the pipeline, the GCN Circulars Archive, and/or published papers.

\section{CATALOG: GCN INFORMATION RETRIEVAL}

After a GRB has triggered Swift or other X-ray $/ \gamma$-ray satellites like INTEGRAL, Fermi, AGILE, or the third Interplanetary Network (IPN), the location of the burst is distributed by the GCN system. Within hours/days, the reports of follow-up observations made by ground-based and space-based optical, 
infrared, radio, and X-ray observers are released through the circulars. If at any time the GRB is observed by Swift, the time of the detection, the most current and most precise position of the afterglow as well as possible information related to its distance (redshift) and the presence of a detected host galaxy are inserted into our Swift Gamma Ray Burst Catalog (see Section 7), available at http://heasarc.gsfc.nasa.gov/cgi-bin/W3Browse/ w3catindex.pl. The trigger time and the position are the essential ingredients to run the Swift data analysis pipeline correctly.

\section{PIPELINE: DATA AND METADATA RETRIEVAL}

The first step in our automated pipeline consists of collecting all the files and metadata useful for the analysis of a specific source (e.g., GRB). Each observation is uniquely identified by an 11 digit number sequence: The first eight digits (the trigger ID) are associated with the GRB and the last three digits refer to different observations of that GRB. Using the trigger ID, the pipeline queries our Swift GRB Catalog for the time and position of the burst, which are used as input for the script (see Section 4). The processing machine has direct, read-only access to the entire Swift archive, which allows the processing script to easily locate the sequence directories for each GRB. In each of these directories the processing script makes use of the unscreened event level 1 data for all the instruments used for the observations of a GRB as well as the UVOT level 1 raw images, if present. In addition, the pipeline uses the attitude and orbit data, the make-filter file, and the spacecraft attitude file from the auxiliary directory and the XRT housekeeping header packets, if the XRT was used for that observation. Only data obtained in the UVOT and XRT pointing modes are included in the analysis. For the XRT, these modes only include photo counting (PC), and window timing (WT) - piled-up mode data are not used (see Hill et al. 2004 for a detailed description of the XRT modes). PC mode is the typical frame transfer operation of a CCD and is used when the sources in the field of view (FOV) have low flux (since the readout time is $2.5 \mathrm{~s}$, sources with a count rate higher than 0.4 counts $\mathrm{s}^{-1}$ will suffer a pile-up effect). Furthermore, PC mode retains full imaging and spectroscopic resolution. In contrast, WT mode loses some of the spatial information: this mode preserves only one-dimensional imaging by binning rows of the CCD and then reading out the central 200 columns of the CCD (corresponding to $8^{\prime}$ ). This enables high resolution timing (the readout time is $2.2 \mathrm{~ms}$ ) with full spectral information for sources with very high count rates.

For every other type of source, such as active galactic nuclei (AGNs), pulsars, etc., the user can specify the location of the directories that contain the level 1 data and the additional files required to run the analysis.

\section{PIPELINE: DATA REDUCTION}

The data reduction step of the pipeline is handled by a single script, swifthlprod (hereafter "the processing script"). This script performs both the data reduction and much of the analysis. It uses the latest release of the HEAsoft software and calibration database, available at the time of analysis. This processing script can be used with a standard HEAsoft distribution and is made available for download at http://heasarc.gsfc.nasa.gov/docs/swift/archive/grbsummary/ software.html.

Before the processing script begins the data reduction in earnest, the attitude file for every observation is corrected for "jumps" in attitude data using the Swift task att jumpcorr. The jumps occur when the attitude is first updated using star tracker information after a slew. The corrected file is then used in the reduction of the XRT and UVOT data.

For all the instruments, the processing script uses standard tools to produce the level 2 data: batgrbproduct for the BAT; xrtpipeline for the XRT; and coordinator, uvotmodmap, uvotimage, uvotbadpix, and uvotexpmap for the UVOT (currently there is not one single tool released by the instrument team that performs the complete data reduction). In the next sections we report in detail the set of parameters used for each tool by swifthlprod and how the user can control the output.

\subsection{BAT}

The standard BAT GRB processing script batgrbproduct is called by swifthlprod to do the data reduction of the event data, assuming the default values, i.e., aperture= "CALDB:FLUX," shortfix="scaledmap,expand," and a partial coding threshold of 5\% (see http://heasarc.nasa.gov/lheasoft/ ftools/headas/batgrbproduct.html for a detailed description of these parameters). The processing script checks the value of the partial coding fraction reported in the output file report.txt, where a summary of the basic analysis results and information about the trigger are given. If this value is smaller than 0.05 (a threshold value indicating that the GRB position is poorly coded) or if batgrbproduct has failed to produce the output directory and files, then the processing script runs batgrbproduct using the aperture parameter fixed at "CALDB:DETECTION" and a minimum partial coding fraction of 0.0. If this trial fails as well, then batgrbproduct has to be run manually, assuming a case by case set of parameters. One example is when batgrbproduct fails to find a burst interval, as may be the case for short, weak GRBs. In this case, the operator must identify the trigger time and the stop time in order to create the standard level 2 products.

\section{2. $X R T$}

Nearly all the data reduction for the XRT PC and WT mode data consists of running the standard script xrtpipeline for each mode with the parameters set to the default values (e.g., for the PC mode, the threshold for the Pulse Height Analyzer, or PHA, of the central pixel is set to 80 and the minimum and maximum PHA values used are 0 and 4095, respectively). Only events with default grades of $0-12$ for PC mode data and $0-2$ for WT mode data are included in the cleaned event files. The processing script runs only the first two stages of xrtpipeline, i.e., it does not generate the standard scientific products. If xrtpipeline fails to produce an output cleaned event file for a particular mode, the data for that sequence/mode combination are not used.

Occasionally, for PC mode data, xrtpipeline is unable to generate a uniform background across the CCD. This is typically due to either contamination by the South Atlantic Anomaly or Earth glow. To check for this, the following steps are taken: (1) the FOV is sub-divided into four quadrants, rotated to align with the PC chip, (2) the background level is estimated in each quadrant, and (3) the background in each quadrant is compared to the mean background of all quadrants. If the ratio of the mean background in any one quadrant to the mean background is larger than a specified threshold (currently set at 1.5 ), the pipeline will stall this sequence and await operator intervention (see also Puccetti et al. 2011 for another method to check for Earth glow). 


\subsection{UVOT}

Since there is no single UVOT data reduction tool provided in HEAsoft, swifthlprod performs the data reduction steps outlined in the UVOT User's Guide v2.2. The UVOT event mode data are minimally processed beyond the standard archive processing. The coordinator tool is run to update the SKY coordinates in the unfiltered event lists using the latest telescope definition and the corrected attitude files. The former contains information about the alignment of the UVOT instrument and the distortion of the image plane and resides in the calibration database. The UVOT image data are checked for whether they have the modulo-8, correlated noise patterns removed. As explained in Section 2.1 of the UVOT User's Guide and in Section 4 of Breeveld et al. (2010), the UVOT spatial resolution is increased because the algorithm on board Swift centroids the events generated by each incoming photon. Since the position of the centroid has a resolution eight times that of the original pixels, the software splits the pixels by a factor of eight to create a raw image. This process creates some artificial fixed-pattern structures that can be clearly seen in the raw images and have to be removed before performing accurate photometry. If any images do not have this correction applied, then the uvotmodmap task is run on them. Both the image and event mode data are then combined into a single multi-extension FITS image for each filter/sequence pair. In the case where an exposure has both event and image data, the former is not included in the analysis.

These multi-extension FITS images are used as input for uvotimage to produce sky images, and next used to calculate the aspect correction. The correction is estimated using, by default, at least five stars in the sky images. If the observation exposure time is too short or there is an insufficient number of bright stars, then the aspect correction is not calculated but must be interpolated from exposures that do have a good aspect correction. Only those exposures that are aspect-corrected are considered in the following analysis. The final steps of the UVOT data reduction are the running of the scripts uvotbadpix and uvotexpmap to create images containing the bad pixels and the exposure maps (for the latter, we leave the parameter method set to "MEANFOV"). As an additional output in the data preparation, the processing script adds all the exposures belonging to each observation in order to improve the signal-tonoise or to detect faint sources using the task uvotimsum.

\section{PIPELINE: DATA ANALYSIS}

All the steps described in this section are performed by swifthlprod. For each instrument, it calls individual tools that extract level 3 products such as light curves, spectra, and sky images. The BAT analysis is done using a custom FTOOLS-like task, batgrbexprod. This task is also made available for download at http://heasarc.gsfc.nasa.gov/docs/swift/archive/grbsummary/ software.html. For the UVOT and XRT analysis, swifthlprod calls the HEAsoft tasks uvotgrblc and xrtgrblc. As explained in Section 6, some of the level 3 products for the XRT require analysis tailored to the specific GRB. This is performed by the pipeline operator using the HEAsoft task xrtgrblcspec. Below, we give a detailed description of how these tasks perform the data analysis.

\subsection{BAT}

\subsubsection{Light Curve}

Among the standard output of batgrbproduct is a set of light curves that cover the full range of event data in the entire
BAT energy band 15-350 keV and in four sub-ranges (15-25, 25-50, 50-150, and 150-350 keV). The time binnings of these light curves are fixed at $1 \mathrm{~s}, 64 \mathrm{~ms}$, and $4 \mathrm{~ms}$. In order to select the best time binning for that particular GRB, batgrbexprod estimates the length $(L)$ of the observation (i.e., defined as the difference between the start and stop times of the total burst interval) and the highest intensity rate $\left(r_{\max }\right)$ observed in the 64 ms light curve. Based on the combination of these two quantities, the best time binning (bin) is chosen as follows. (1) If $r_{\max } \geqslant 1$ then bin $=2 \mathrm{~ms}(L<2.5 \mathrm{~s}), 64 \mathrm{~ms}(2.5 \leqslant L<$ $100 \mathrm{~s})$, and $256 \mathrm{~ms}(L \geqslant 100 \mathrm{~s})$. (2) If $r_{\max }<1$ then bin $=16 \mathrm{~ms}$ $(L<2.5 \mathrm{~s}), 256 \mathrm{~ms}(2.5 \leqslant L<100 \mathrm{~s})$, and $1 \mathrm{~s}(L \geqslant 100 \mathrm{~s})$. Since batgrbproduct does not produce light curves with a binning of 16 and $256 \mathrm{~ms}$, batbinevt is used to produce the light curves for the entire energy band and the four sub-ranges.

\subsubsection{Spectrum}

Spectra and response matrices are part of the standard output of batgrbproduct. Among others, spectra are computed for the total burst interval and the pre-slew, slew, and post-slew intervals. The response matrices are generated only for the preslew and post-slew intervals, since the BAT energy response generator, batdrmgen, works for a fixed single incident angle of the source, i.e., for the pre-slew and post-slew intervals, when the spacecraft is not slewing. For the slewing interval, we follow the method explained in Sakamoto et al. 2008: in summary, batgrbexprod (1) divides the interval into sub-intervals, each $5 \mathrm{~s}$ long (if the last bin is shorter than $2 \mathrm{~s}$, then it is appended to the second to last bin), (2) runs batbinevt to generate the PHA file corresponding to the bin, (3) updates the keywords of the PHA file (batupdatephakw), and (4) creates the response matrices with batdrmgen, taking into account the position of the GRB in detector coordinates. All the newly produced matrices are weighted and combined to create an average response matrix for the slewing interval using addrmf. The weighting factors for each matrix are estimated as follows: $\operatorname{bin}_{c} / \operatorname{tot}_{c}$, where $\operatorname{bin}_{c}$ and $\operatorname{tot}_{c}$ are the number of counts in each bin and in the entire slew interval. The spectra of the pre-slew, slew, and post-slew intervals are fitted within Xspec with two models, a simple power law and a cutoff power law (see Sakamoto et al. 2008 for their definition). As explained in Sakamoto et al. (2008), a $\Delta \chi^{2}>6$ is the criterion to select the cutoff power law over the simple power law.

A similar approach to creating files for a spectral analysis is adopted to convert the Bayesian block light curve into units of erg $\mathrm{cm}^{-2} \mathrm{~s}^{-1}$. The conversion is obtained extrapolating the 15-150 keV mask weighted (background subtracted) BAT light curve into the XRT $0.3-10 \mathrm{keV}$ (and 2-10 keV) energy band. The selected light curve has Bayesian block bins and is part of the batgrbproduct standard output. This conversion compares the temporal source behavior covered by BAT and XRT observations over a long period of time. The conversion is based on the assumption that there is one continuous spectral behavior (a power law) over the $0.3-150 \mathrm{keV}$ spectral range. Since the XRT and the BAT do not share a common energy range, this assumption had to be tested (Sakamoto et al. 2007). The choice of the $2-10 \mathrm{keV}$ range is due to the fact that it is the XRT range closer to the BAT energy window. The Bayesian block input light curve can have bins that cross the time boundaries of the pre-slew, slew, and post-slew intervals (e.g., part of the block is in one interval and the remaining in another interval). The PHA file and its response function corresponding to this bin has to be created accordingly, following the 
Table 1

XRT Extraction Regions

\begin{tabular}{|c|c|c|c|c|c|}
\hline \multicolumn{3}{|c|}{$\mathrm{PC}$} & \multicolumn{3}{|c|}{ WT } \\
\hline $\begin{array}{l}\text { Rate } \\
\text { (1) }\end{array}$ & $\begin{array}{l}\text { GRB } \\
\text { (2) }\end{array}$ & $\begin{array}{l}\text { BKG } \\
\text { (3) }\end{array}$ & $\begin{array}{l}\text { Rate } \\
(4)\end{array}$ & $\begin{array}{c}\text { GRB } \\
(5)\end{array}$ & $\begin{array}{l}\text { Pile-up } \\
\text { (6) }\end{array}$ \\
\hline $\mathrm{c} / \mathrm{s}>7$ & $\mathrm{~A}\left(20^{\prime \prime}, 105^{\prime \prime}\right)$ & $\mathrm{A}\left(130^{\prime \prime}, 260^{\prime \prime}\right)$ & $\mathrm{c} / \mathrm{s}>1000$ & $\mathrm{~B}\left(450^{\prime \prime}\right)$ & $\mathrm{B}\left(37^{\prime \prime} .84\right)$ \\
\hline $5<\mathrm{c} / \mathrm{s} \leqslant 7$ & $\mathrm{~A}\left(15^{\prime \prime}, 95^{\prime \prime}\right)$ & $\mathrm{A}\left(120^{\prime \prime}, 240^{\prime \prime}\right)$ & $600<\mathrm{c} / \mathrm{s} \leqslant 1000$ & $\mathrm{~B}\left(450^{\prime \prime}\right)$ & $\mathrm{B}\left(28^{\prime \prime} .38\right)$ \\
\hline $3<\mathrm{c} / \mathrm{s} \leqslant 5$ & $\mathrm{~A}\left(12^{\prime \prime}, 85^{\prime \prime}\right)$ & $\mathrm{A}\left(110^{\prime \prime}, 220^{\prime \prime}\right)$ & $400<\mathrm{c} / \mathrm{s} \leqslant 600$ & $\mathrm{~B}\left(450^{\prime \prime}\right)$ & $\mathrm{B}\left(23^{\prime \prime} .65\right)$ \\
\hline $1<\mathrm{c} / \mathrm{s} \leqslant 3$ & $\mathrm{~A}\left(10^{\prime \prime}, 75^{\prime \prime}\right)$ & $\mathrm{A}\left(100^{\prime \prime}, 200^{\prime \prime}\right)$ & $300<\mathrm{c} / \mathrm{s} \leqslant 400$ & $\mathrm{~B}\left(300^{\prime \prime}\right)$ & $\mathrm{B}\left(9^{\prime \prime} 43\right)$ \\
\hline $0.5<\mathrm{c} / \mathrm{s} \leqslant 1$ & $\mathrm{~A}\left(5^{\prime \prime}, 65^{\prime \prime}\right)$ & $\mathrm{A}\left(90^{\prime \prime}, 190^{\prime \prime}\right)$ & $100<\mathrm{c} / \mathrm{s} \leqslant 300$ & $\mathrm{~B}\left(236^{\prime \prime}\right)$ & $\mathrm{B}\left(0^{\prime \prime}\right)$ \\
\hline $0.1<\mathrm{c} / \mathrm{s} \leqslant 0.5$ & $\mathrm{C}\left(55^{\prime \prime}\right)$ & $\mathrm{A}\left(80^{\prime \prime}, 185^{\prime \prime}\right)$ & $50<\mathrm{c} / \mathrm{s} \leqslant 100$ & $\mathrm{~B}\left(200^{\prime \prime}\right)$ & $\mathrm{B}\left(0^{\prime \prime}\right)$ \\
\hline $0.05<\mathrm{c} / \mathrm{s} \leqslant 0.1$ & $\mathrm{C}\left(45^{\prime \prime}\right)$ & $\mathrm{A}\left(65^{\prime \prime}, 165^{\prime \prime}\right)$ & $10<\mathrm{c} / \mathrm{s} \leqslant 50$ & $\mathrm{~B}\left(165^{\prime \prime}\right)$ & $\mathrm{B}\left(0^{\prime \prime}\right)$ \\
\hline $0.01<\mathrm{c} / \mathrm{s} \leqslant 0.05$ & $\mathrm{C}\left(25^{\prime \prime}\right)$ & $\mathrm{A}\left(40^{\prime \prime}, 140^{\prime \prime}\right)$ & $5<\mathrm{c} / \mathrm{s} \leqslant 10$ & $\mathrm{~B}\left(118^{\prime \prime}\right)$ & $\mathrm{B}\left(0^{\prime \prime}\right)$ \\
\hline $0.005<\mathrm{c} / \mathrm{s} \leqslant 0.01$ & $\mathrm{C}\left(22^{\prime \prime}\right)$ & $\mathrm{A}\left(40^{\prime \prime}, 140^{\prime \prime}\right)$ & $1<\mathrm{c} / \mathrm{s} \leqslant 5$ & $\mathrm{~B}\left(71^{\prime \prime}\right)$ & $\mathrm{B}\left(0^{\prime \prime}\right)$ \\
\hline $0.001<\mathrm{c} / \mathrm{s} \leqslant 0.005$ & $\mathrm{C}\left(17^{\prime \prime}\right)$ & $\mathrm{A}\left(40^{\prime \prime}, 140^{\prime \prime}\right)$ & $\mathrm{c} / \mathrm{s} \leqslant 1$ & $\mathrm{~B}\left(35^{\prime \prime}\right)$ & $\mathrm{B}\left(0^{\prime \prime}\right)$ \\
\hline$c / s \leqslant 0.001$ & $\mathrm{C}\left(12^{\prime \prime}\right)$ & $\mathrm{A}\left(40^{\prime \prime}, 140^{\prime \prime}\right)$ & & & \\
\hline
\end{tabular}

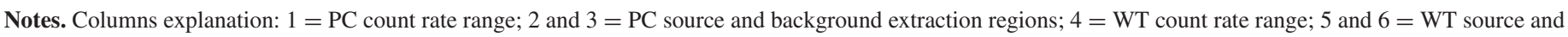
pile-up extraction regions. The region shapes are: $\mathrm{A}=$ annulus, $\mathrm{B}=\mathrm{box}, \mathrm{C}=$ circle.

method described above. For each bin, the spectral analysis is performed within Xspec, assuming a simple power law model. The count rate and fluxes in the $0.3-10 \mathrm{keV}$ and $2-10 \mathrm{keV}$ energy bands are estimated and used for the conversion. If a good fit cannot be found for a given Bayesian block, then this block is merged with the previous block, and the fitting process is repeated. The errors in each BAT light curve bin were found by randomly sampling the fitted model parameters within their respective confidence intervals 1000 times, and recalculating the flux each time. The $1 \sigma$ confidence interval in this flux conversion sample is used as the error. Evans et al. (2010) describe a somewhat different approach for combined XRT/BAT analysis, including a discussion of the many caveats that need to be considered when extrapolating from one instrument to another with a different bandpass. The results of this analysis for the growing list of Swift GRBs are available at the Web site http://www.swift.ac.uk/burst_analyser/.

\section{2. $X R T$}

Once the level 2 products are created following the processing described in Section 4.2, the task xrtgrblc determines the start and stop times of each orbit using the make-filter file stored in the auxiliary directory. This file contains selected spacecraft information related to the observation. The task considers as an orbit the time interval in which the XRT CCD is active (i.e., when the reference voltage for the variable "amp 1 " is read from the filter file), the angular distance from the target is less than $10^{\prime}$, the spacecraft is settled on the target, and when the gap between two consecutive X-ray photons reaching the CCD is less than $100 \mathrm{~s}$. At this stage the data preparation follows one of the following two paths, depending on the mode of the observation.

1. WT mode. Despite having selected only times where the spacecraft is considered settled, some small adjustments in the pointing can happen, in particular, at the very beginning of the observation, when the observation mode is most likely to be WT. The orbit boundaries are modified in order to consider only those times when the spacecraft roll angle is less than $1^{\circ}$ from the final settle point.

2. PC mode. Before the light curve extraction and analysis begins, the location and intensity of any field sources in the FOV are determined using Ximage. These two quantities define the size and location of regions that must be excluded from the light curve analysis.

\subsubsection{Light Curve}

To have a fully corrected XRT light curve, adjustments have to be considered to address some issues, e.g., the extreme source variability, the pile-up effect for bright sources, and some CCD artifacts (Evans et al. 2009). The variability and the pile-up effects can be taken care of by selecting the correct size of the source, background, and pile-up extraction regions. The selection is dependent on the event data mode, and the two modes (PC and WT) each warrant their own discussion. For the PC mode a preliminary light curve for each orbit from a $40^{\prime \prime}$ circle centered on the source is extracted using the FTOOLS extractor and with a time binning of $20 \mathrm{~s}$. A sliding-cell-type algorithm is applied to this preliminary light curve to determine the time-dependent source and background extraction regions, and any pile-up exclusion. This algorithm uses the running weighted mean count rate to divide the light curve into time intervals during which the count rate falls within empirically determined ranges. Each count rate range has a corresponding source and background region shape. Using this procedure, during periods of high count rate, larger source and background regions are selected. The regions for the source are either circular or annular (the latter to avoid pixels affected by pile-up), while the background regions are always source-centered annuli. The region size for the source (GRB) and background (BKG) are reported in the left section of Table 1.

The source regions were selected to maximize the contribution of the source and to minimize the number of background photons. A reverse approach has been adopted to determine the background region size, with the additional caveat of considering areas relatively close to the source. Circular regions associated with field sources are subtracted from both the source and background regions. Their radii correspond to twice the halfbox length reported by Ximage. If the field source is within $0^{\prime} \cdot 6$ of the source, then this radius is reduced by half.

Sometimes the source position is close to the edges instead of the center of the CCD. For GRBs, this will typically happen when the detection position is not well known, e.g., when the GRB is discovered by missions that carry instruments with poor spatial resolution, like Fermi or the IPN. In this case, the size of the source extraction region will be reduced from the empirical values of Table 1 in order to be fully contained in the CCD. 
The background region will then be a circle of $150^{\prime \prime}$ located close to the source, fully contained in the CCD, and with field sources excluded.

For the WT mode event data, both the preliminary and final light curve extraction regions are positioned over the WT strip using a combination of the HEAsoft tools pointxform, sky2xy and xy2sky, and the spacecraft housekeeping data. The regions themselves are all 20" wide. Similar to the PC mode, a preliminary light curve is extracted for each orbit from a 50" long box centered on the source using the FTOOLSextractor and with a time binning of $4 \mathrm{~s}$. The above sliding-cell algorithm is applied to this preliminary light curve. As shown in Romano et al. (2006), the pile-up effect can be present for very bright sources. Following their results, we selected as source and pileup regions, boxes centered on the source position and with length as a function of the counts rate. The sizes of these boxes are reported in Table 1 . The background regions are a $450^{\prime \prime}$ long box centered on the WT strip, excluding the source region plus an additional $55^{\prime \prime}$ on either side to avoid point-spread function (PSF) contamination. Field sources are ignored in the WT mode.

The task xrtgrblc extracts light curves in the $0.3-10 \mathrm{keV}$, $0.3-2 \mathrm{keV}$, and 2-10 keV energy bands. These curves are uniformly binned and the bin duration used is $0.01766 \mathrm{~s}$ for the WT mode and $2.51 \mathrm{~s}$ for the PC mode. The source light curve must be corrected for the presence of pile-up, irregularities in the exposure map (e.g., bad CCD pixels and columns, and attitude variations), and the PSF. The correction factor is estimated using the HEAsoft tool xrtlccorr. The background light curve is corrected only for irregularities in the exposure map using the HEAsoft tools xrtexpomap and Ximage. The output of this is subtracted from the raw source light curve and then multiplied by the total source correction factor to give the net corrected light curve. The total PC and WT corrected light curves are binned using a step-binning algorithm, where the number of counts included in each bin is proportional to the average rate in that bin. The algorithm requires that each bin has at least 20 counts and a signal-to-noise ratio of at least 3 . Often at the end of the PC light curve, there are bins that do not satisfy these conditions. In this case, a $3 \sigma$ Bayesian upper limit is calculated. Since the WT mode is active only for high-count-rate epochs, low signalto-noise bins are not converted to upper limits and are discarded from the binned light curve. This method is also applied to the hard (2-10 keV) band. The hard band bin boundaries are applied to the soft $(0.3-10 \mathrm{keV})$ band, and a hardness ratio, defined as hard vs. soft, is calculated.

A source and background spectrum for each of the timedependent extraction regions found previously are extracted. The background-subtracted source spectrum in the $0.3-10 \mathrm{keV}$ energy range is fitted with a doubly absorbed power law. One of the two absorption components is fixed to the Galactic value and the other is left free to vary. This spectral fit is necessary to obtain the flux conversion factors in the $0.3-10 \mathrm{keV}, 0.3-2 \mathrm{keV}$, and $2-10 \mathrm{keV}$ energy ranges. If the fit is $\operatorname{good}\left(0.7 \leqslant \chi_{r}^{2} \leqslant 1.3\right)$, the conversion factor is estimated as the ratio of the model flux to the count rate. If not, then the flux conversion factor from the previous interval is used. If there is no prior good fit, then mean source flux conversion factors of $5 \times 10^{-11} \mathrm{erg} \mathrm{cm}^{-2}$ count $^{-1}$ $(0.3-10 \mathrm{keV}), 2.7 \times 10^{-11} \mathrm{erg} \mathrm{cm}^{-2}$ count $^{-1}(0.3-2 \mathrm{keV})$, and $2.3 \times 10^{-11} \mathrm{erg} \mathrm{cm}^{-2}$ count $^{-1}(2-10 \mathrm{keV})$ are used.

The script xrtgrblcspec calls Xspec to fit the corrected 0.3-10 keV light curve with three models that reproduce the typical trends observed in GRB light curve decay: a simple, a broken, and a double broken power law. The best fit is found using a series of $F$-tests, with a significance threshold of $3 \sigma$. If there is some GRB flaring activity, the above models will not adequately represent the light curve. For this reason, we always visually inspect the light curve fits and manually remove flares if necessary. By doing so, we ensure that the fit only reproduces the continuum light curve. The human intervention also ensures that the fit is as optimal as possible. There are a few cases where the light curve should be fitted with a more complex model than the double broken power law; Evans et al. (2009) found that 110 light curves out of 665 required more than two breaks of $4 \sigma$. Unfortunately, Xspec does not provide a power law model with a variable number of breaks. In those cases, the user tries to minimize the residual by using the double broken power law model.

\subsubsection{Spectrum}

The breaks in the XRT light curve can emphasize a change in the environment where the X-rays are emitted or a change in the physical properties of the emitting region. We use these breaks to define time intervals where we expect to find no spectral variability. If the light curve is best fitted with a simple power law, the entire observation is considered as a single interval. xrtgrblc automatically sums all the source 0.3-10 keV PHA files and weighted ancillary response functions (ARFs) for the orbits that belong to each time interval in the light curve. The ARFs are generated using the xrtmkarf task (Moretti et al. 2005). The sum is obtained running the FTOOLS tasks mathpha and addarf (the error method used when calling the mathpha task is Gaussian). The reason for summing the PHA file is twofold: (1) the number of orbits can be large enough to create problems for Xspec in fitting each orbit as a single data set and (2) the better signal-to-noise makes it easier to see spectral features in the combined spectrum than in the individual spectra. The combined spectra are fitted using a double absorbed power law, where one absorption is fixed at the Galactic value and the other is left free to vary. The spectral analysis can reveal a change in the level of absorption or in the intrinsic photon distribution.

\subsection{UVOT}

The task uvotgrblc uses uvotsource to extract photometric light curves from UVOT images. The accuracy of these curves is ensured by applying the coincidence-loss and largescale sensitivity correction (estimated by uvotsource) and by accounting for several known issues: (1) presence of contaminating field sources, (2) selection of the background extraction region, (3) significance of the detection, (4) position, and (5) size of the source aperture. These issues are addressed as follows.

1. Field sources and background region. The choice of the background region depends on two factors: the presence of field sources (i.e., their intensity and their location with respect to the source) and the intensity of the source (i.e., how far the PSF wings extend). The first step is to run uvotdetect to obtain a list of field sources and their exclusion regions in each UVOT summed image. Next, uvotgrblc selects the optimal region size by estimating the background of the summed images in three annular regions centered on the position of the source (in the case of our analysis of GRBs, it is derived from our Swift GRB Catalog). The annuli have inner-outer radii of $15-27^{\prime \prime}$, $27-35^{\prime \prime}$, and 35-42" , respectively, and detected field sources are excluded. We are confident that the choice of these 
radii is conservative and no significant contribution of the source PSF is expected, at least in the largest annulus (see Figure 5 in Poole et al. 2008, where it is shown that the wings of the PSF can be seen up to a maximum of $25^{\prime \prime}$ from the source center). The three background values are compared to each other and the region with the lowest value is selected. There is a chance that all three regions were contaminated by photons from a nearby source (e.g., a galaxy) or CCD artifacts (e.g., ghost images or readout streaks). The background region is visually inspected for these types of contaminants. If there is a problem, a custom background region is selected and the processing script is run again for this source.

2. Significance of detection. An approximate significance is initially determined by extracting the number of counts in a $3^{\prime \prime}$ radius circle and in the background region (see above) for all the exposures in every filter. For dim sources, the source aperture can be background-dominated. Thus, we consider as a detection every time $\sigma_{\text {det }}>3$. We use $\sigma_{\text {det }}=$ counts $_{\text {net }} / \sigma_{\text {tot }}$, where we define $\sigma_{\text {tot }}=\left(\sigma_{\text {Source }}^{2}+\sigma_{\text {bkg }}^{2}\right)^{1 / 2}$. In

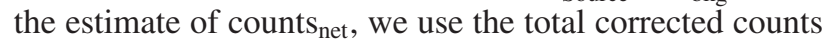
if the counts frame ${ }^{-1}$ is $>1$, and the total corrected counts scaled by a factor of

$$
1-\frac{\text { total counts }}{\text { number of frames }}
$$

otherwise. This is a conservative approach that does not allow us to miss weak sources.

3. Aperture position. The extraction region must be centered exactly on the source. If there is a detection in any filter (see above), the coordinates of the object closest to the position provided by the user (or by the Swift GRB Catalog in our analysis) are refined using the task uvotcentroid. The task is run on the exposures with the highest detection level for each filter. The average right ascension (R.A.) and declination (decl.) are found after a single round of $1 \sigma$ rejection of outliers. If the new position is beyond $3^{\prime \prime}$ from the catalog position, then no adjustment is made and the catalog's R.A. and decl. are used. If the new coordinates are offset from the inputs by more than $1 . .5$, then the initial estimates of the significance of detection in each exposure are recalculated, as well as the optimal background regions.

4. Aperture size. The standard photometric aperture is a circle with a 5" radius (Poole et al. 2008). This area is large enough to collect most photons for a moderately bright object. For weaker objects, the PSF wings are too dim and the area is mostly background-dominated: the radius of the source extraction region must change accordingly to determine the significance of detection and to extract the best photometric information. The intensity and position of the field sources, combined with the initial source intensities, are used to determine the optimal size for the photometry aperture. The selection of the aperture radius is as follows: (1) $5^{\prime \prime}$ if the nearest neighbor is distant $\left(>15^{\prime \prime}\right)$ or is closer $\left(>10^{\prime \prime}\right)$ but $\operatorname{dim}(<0.1 \mathrm{c} / \mathrm{s}),(2) 4^{\prime \prime}$ if the nearest neighbor is closer $\left(>10^{\prime \prime}\right)$ but brighter $(0.1<\mathrm{c} / \mathrm{s} \leqslant 10)$, (3) fixed at $3^{\prime \prime}$ for all other cases. The radius can be reduced from $5^{\prime \prime}$ and $4^{\prime \prime}$ to smaller dimensions based on the source intensity, if detected: for $\mathrm{c} / \mathrm{s}<0.1$, the radius becomes $3^{\prime \prime}$; for $0.1<$ $\mathrm{c} / \mathrm{s}<0.75$ the radius is fixed at $4^{\prime \prime}$.

If the source extraction region is smaller than $5^{\prime \prime}$ then the photometric estimates derived using uvotsource have to be aperture-corrected. To estimate the correction factor, the three "best" field sources for every OBS_ID are selected. Each source must meet the following selection criteria to be considered a good candidate for aperture correction: (1) be nearly circular in shape according to uvotdetect (i.e., the ratio of the semimajor to semiminor axes is less than $1.5)$, (2) lie in the range of $20^{\prime \prime}$ to $200^{\prime \prime}$ from the source, (3) have a size $<7^{\prime \prime}$, (4) have a count rate in the range of $0.5 \mathrm{c} / \mathrm{s}$ to $5 \mathrm{c} / \mathrm{s}$, (5) not have any nearby neighbors, and (6) have an average exposure $>95 \%$ of the summed image exposure. The ratio of the count rates obtained in circular regions with $5^{\prime \prime}$ radius and with $3^{\prime \prime}$ or $4^{\prime \prime}$ gives the aperture correction factor for each of the three stars. The pipeline selects as optimal the median of the three values, limiting the possibility of error in the selection of the three objects. If three good sources cannot be found by this method, then the aperture correction is estimated using the parameter apermeth=CURVEOFGROWTH in the task uvotsource.

If the source was not detected in any consecutive images, those images are summed and photometry is performed on the result. If in any image, or summed image, no source is detected, an upper limit is estimated. The photometric measurements and the upper limits are derived from the FLUX_HZ (and FLUX_HZ_ERR) and FLUX_HZ_LIM columns, respectively, in the uvotsource output FITS file (obtained with the parameter apercorr set to NONE). They are expressed in units of flux density and are not suitable to generate a combined light curve in which all the filters are compared. All these quantities can be converted to units of flux ( $\left.\mathrm{erg} \mathrm{cm}^{-2} \mathrm{~s}^{-1}\right)$. The formulas used to estimate the flux for the detection, its error, and for the upper limit are the following:

$$
\begin{aligned}
& \text { FLUX }_{\text {Det }}=\text { FLUX_HZ } \times \text { AREA } \times \text { APERTURE } \times 1 E-26 \\
& F \text { AUX_ERR } \\
& \text { FLet }=\text { FLUX_HZ_ERR } / \text { FLUX_HZ } \times \text { FLUX } \\
& \text { Det } \\
& \text { FLUX_HZ_LIM } \times \text { AREA } \times 1 E-26,
\end{aligned}
$$

where APERTURE is the correction factor, and AREA is the integrated filter response convolved with a power-law spectrum with index $\alpha=1$. The functions that reproduce the filter response can be found in the CALDB files swuXX_20041120v10Y.arf, where $\mathrm{XX}$ indicates the filter and $\mathrm{Y}$ the version. Since all the flux densities are multiplied by the filter response, the integrated flux light curve will present the white light curve above all the other filters, due to its larger band pass (e.g., see Figure 12, right panel).

\section{PIPELINE: OUTPUT PRODUCTS}

From the level 3 products (light curves, spectra, images) and the results of the temporal and spectral analyses, a series of Web pages are automatically generated for each source. The typical products for the three instruments are gif images and FITS files. The images show a small area of the sky around the source direction, the light curves, and the plots of the spectral analysis (for the BAT and the XRT only). The FITS files can be divided into three main categories: light curves, spectral files (PHA, ARF, and response matrix files or RMF), and a summary file for the BAT and XRT instruments. The summary file contains all the results of the light curve and/or the spectral analysis, together with the indication of important quantities used in the data analysis. These quantities are used to populate various tables contained in the Web pages. The files produced by our pipeline are available for download from the HEASARC archive Web site: http://heasarc.gsfc.nasa.gov/docs/swift/archive/grbsummary/ grb_pages/20XX/grbXXXXXXX/. For an explanation of the 


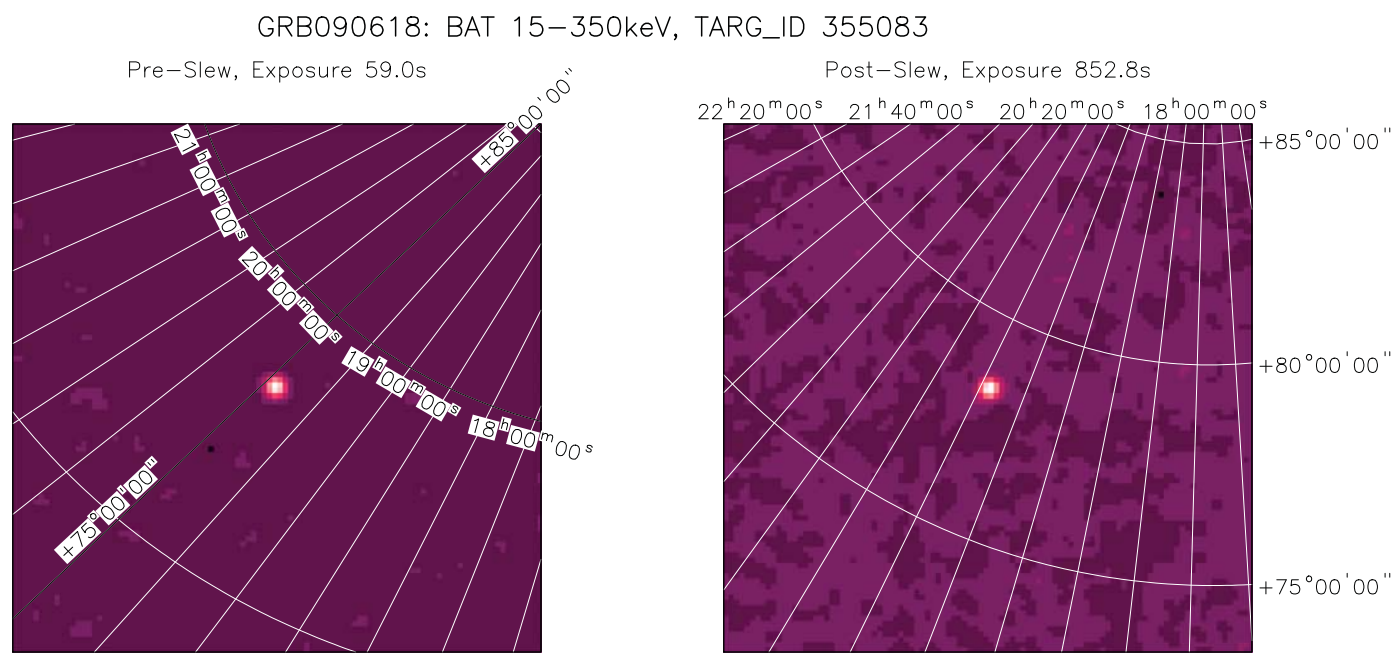

Figure 1. BAT 15-350 keV FOV in the pre-slew and post-slew intervals.

(A color version of this figure is available in the online journal.)

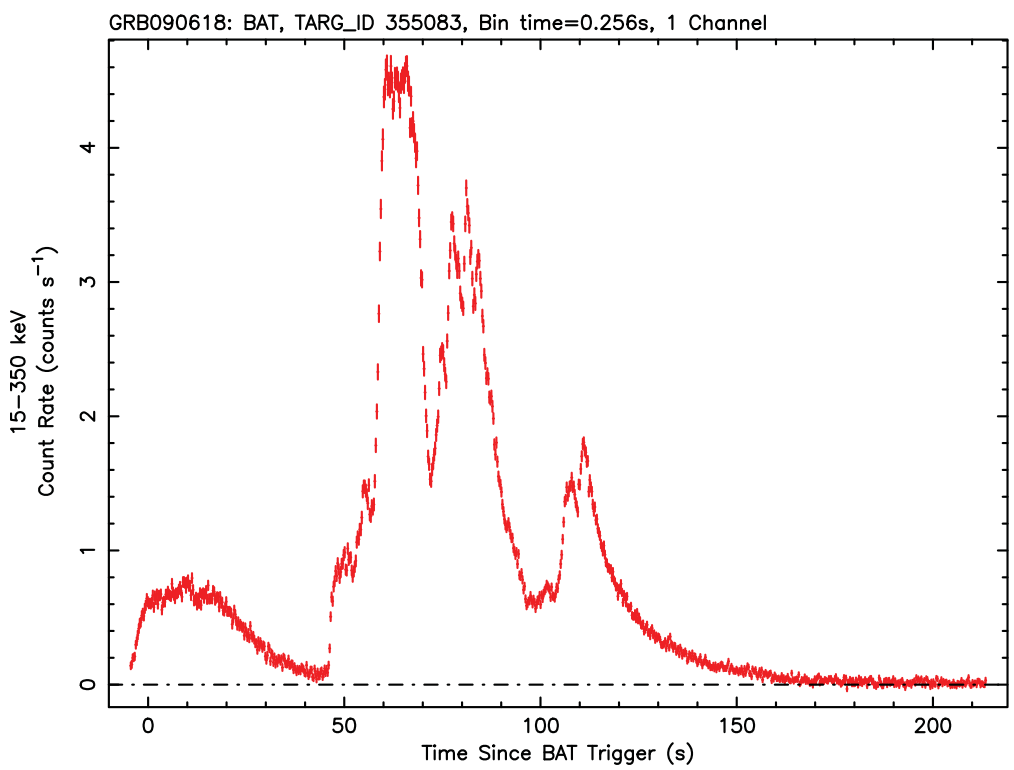

Figure 2. BAT 15-350 keV background subtracted light curve.

(A color version of this figure is available in the online journal.)

naming convention adopted for the files, see the document at http://heasarc.gsfc.nasa.gov/docs/swift/archive/grbsummary/ Filenames.pdf. A detailed description of all the available products is given below.

\subsection{BAT}

\subsubsection{Images}

The script batgrbproduct generates three sets of gif images for the BAT instrument and they provide visual information of the spatial, temporal, and spectral analyses. The three sets contain the following:

1. The FOV of the detected burst derived from the event data during the pre-slew and, if available, after the slew centered on the GRB position. Both images, standard products of the task batgrbproduct, show the source in the $15-350 \mathrm{keV}$ energy range and are background-subtracted (Figure 1).

2. The mask-weighted light curves of the full GRB duration (T100, that is, the time interval from the emission start time to the emission end time). T100 is determined by battblocks using a Bayesian-block technique (Sakamoto et al. 2011). The light curves are obtained with the optimal time binning in the total 15-350 keV light curve (Figure 2) and in four energy ranges, corresponding to 15-25, 25-50, 50-100, and 100-350 keV (Figure 3). Both plots have an additional $5 \%$ of the T100 interval at both boundaries for plot purposes. The $y$-axis unit is counts $\mathrm{s}^{-1} \operatorname{det}^{-1}$, where a det is $0.4 \times 0.4=0.16 \mathrm{~cm}^{2}$, and the time binning is chosen based on the length and intensity of the burst (see Section 3). In the second plot, the slew interval, if present, is marked with a horizontal line.

3. The results of the spectral fit with a power law and cutoff power law in the $15-150 \mathrm{keV}$ range for each epoch, i.e., pre-slew, slew, post-slew, and total (Figure 4).

\subsubsection{FITS}

The FITS files created by batgrbproduct, used to generate the images described above and to summarize the spectral analysis, are the following: 


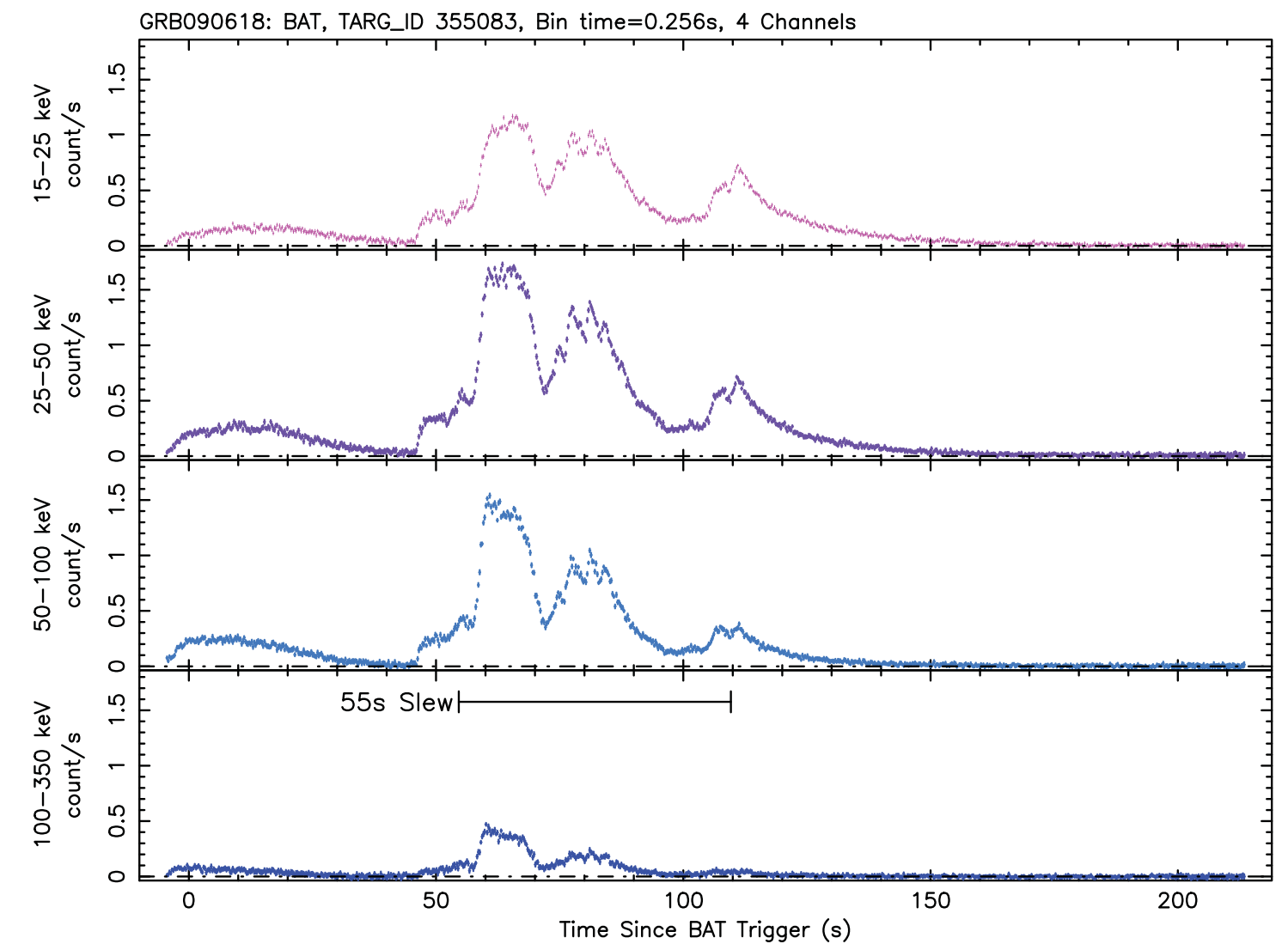

Figure 3. BAT background-subtracted light curves in the four energy ranges (15-25, 25-50, 50-100, and 100-350 keV).

(A color version of this figure is available in the online journal.)
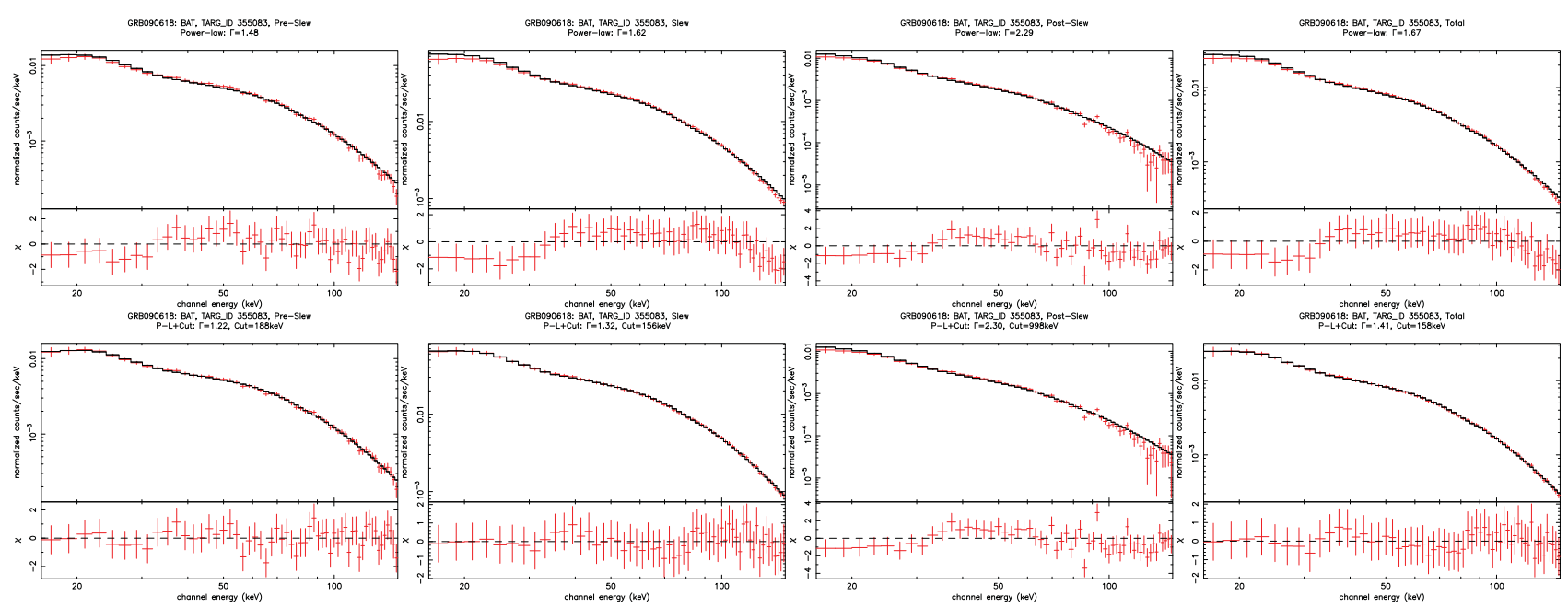

Figure 4. Spectral fit of the BAT pre-slew, slew, post-slew, and the total intervals with the simple power law (top) and power law with cutoff (bottom).

(A color version of this figure is available in the online journal.)

1. The fixed time bin standard one- and four-channel light curves as produced by batbinevt.

2. The Bayesian binned light curves (see Section 5.1) in units of erg $\mathrm{cm}^{-2} \mathrm{~s}^{-1}$ in the ranges $0.3-10,2-10,15-25,25-50$, $50-100,100-150$, and $15-150 \mathrm{keV}$. For each bin, the values of the spectral fit and the statistic are given.

3. A PHA spectrum and corresponding response matrix for each epoch (i.e., pre-slew, slew, post-slew, and total).

4. The information file (BATINFO extension), containing all the values of the spectral analysis, e.g., spectral index of the power law (with errors) and its normalization, cutoff energy (with errors), model flux in various energy ranges, degrees of freedom, and $\chi^{2}$ value.

\section{2. $X R T$}

\subsubsection{Images}

Similarly to the BAT, there are three sets of gif images for the XRT. The script xrtgrblc generates the following:

1. The $0.3-10 \mathrm{keV}$ images centered on the source with a large and a small FOV. In both modes the plots show the extraction regions for the source (in solid blue) and the 
XRT $0.3-10.0 \mathrm{keV}$

GRB090618

TARG_ID 355083

Exposure $112.4 \mathrm{~s}$

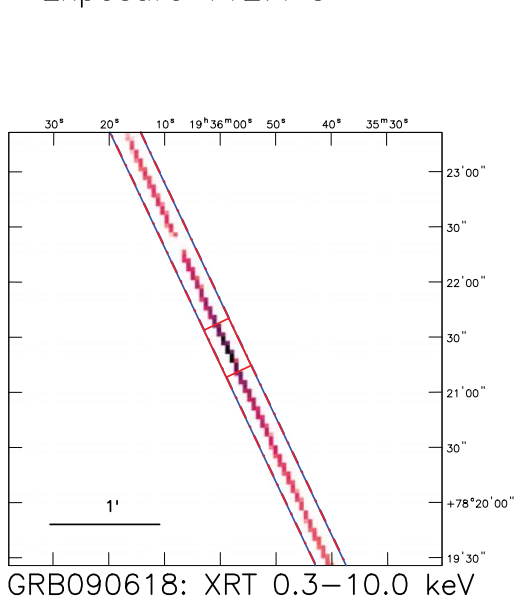

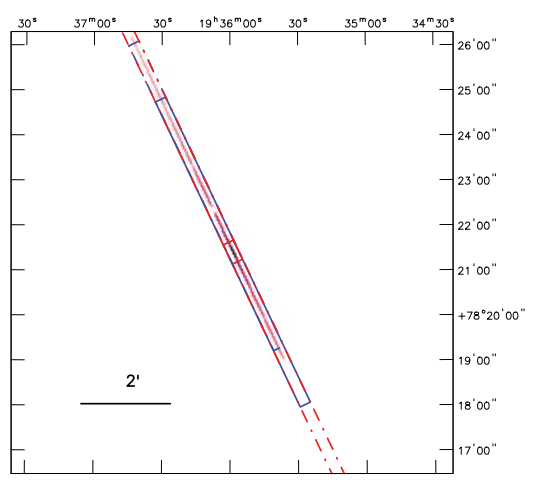

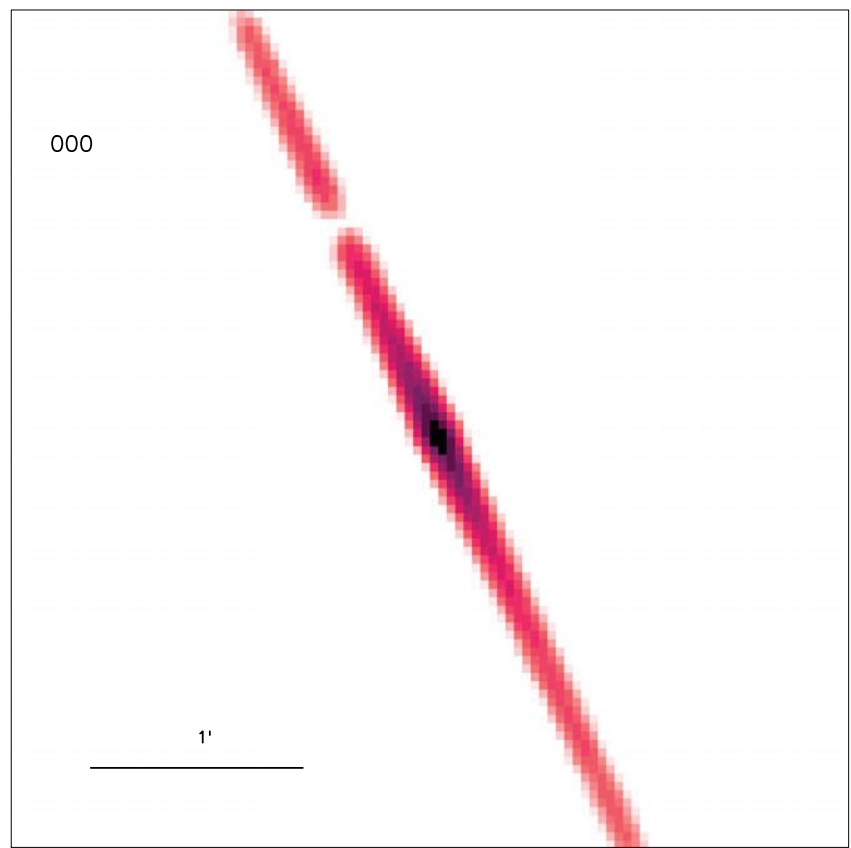

Figure 5. Top: 0.3-10 keV WT mode large and small FOVs of the first observation. Bottom: $0.3-10 \mathrm{keV}$ WT mode small FOV of all the orbits for all the observations combined.

(A color version of this figure is available in the online journal.)

background (in dashed blue) and the excluded areas (in dashed red), such as the field sources in the PC mode, and the pile-up region in the WT mode). The PC image is the sum of all the observations (Figure 5, upper panel), while the WT image refers to the interval with the highest signalto-noise ratio (Figure 6, upper panel).

2. A mosaic of the images of each single observation ID, to show the detection and variability of the source during the entire observational campaign (Figure 5 and Figure 6, lower panels, for WT and PC mode, respectively).

3. The $0.3-10 \mathrm{keV}$ combined WT and PC binned light curves in units of count rate (Figure 7, upper panel). The position in time when the shape of the source ex- traction regions change are indicated by up arrows. The blue and red colors are adopted for the WT mode and the PC mode, respectively. At the top of the plot the OBS_ID time boundaries are shown. The bottom part of the plot reproduces the fractional exposure of each light curve bin.

4. The $0.3-10 \mathrm{keV}$ combined WT and PC binned light curves in units of erg $\mathrm{cm}^{-2} \mathrm{~s}^{-1}$ (Figure 7, lower panel). The procedure to estimate the conversion factors from rate to flux is explained in Section 5.2.

5. The combined WT and PC hardness ratio, defined as the ratio of the $2-10 \mathrm{keV}$ to $0.3-2 \mathrm{keV}$ binned light curves (Figure 8). 
XRT $0.3-10.0 \mathrm{keV}$

GRB090618

TARG_ID 355083

Exposure $230866.3 \mathrm{~s}$
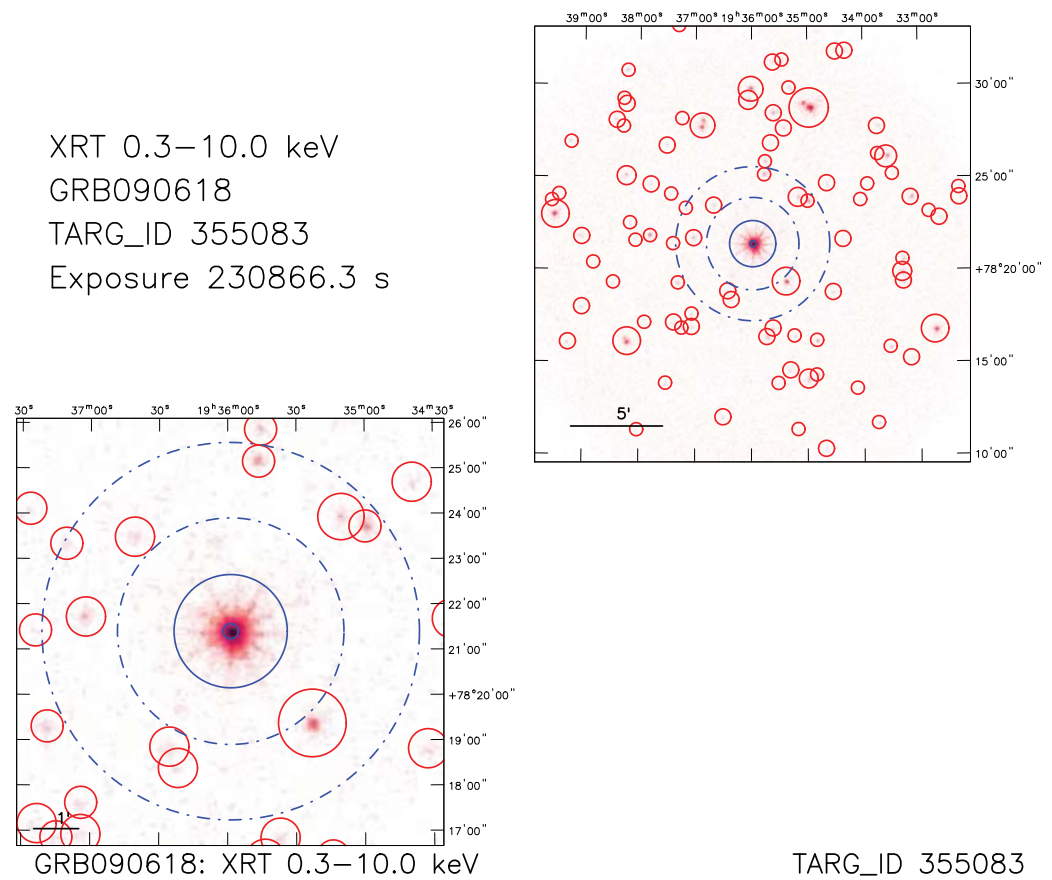
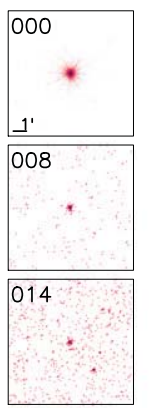

020.

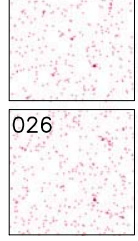

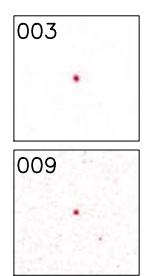
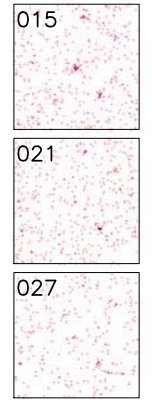
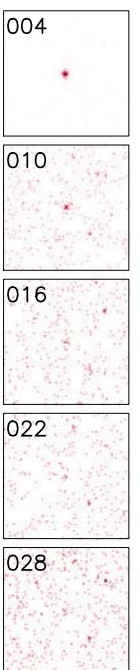
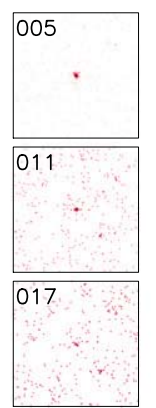

023

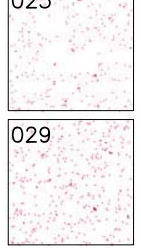

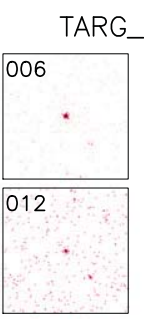
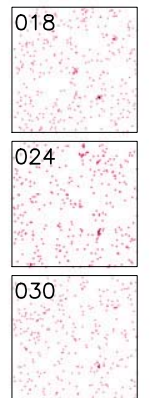

355083
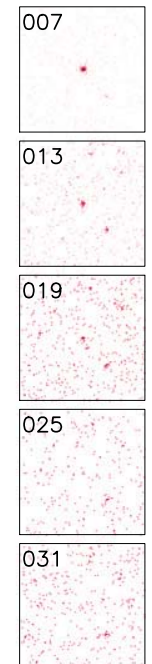

Figure 6. Top: 0.3-10 keV PC mode large and small FOVs of the first observation. Bottom: $0.3-10 \mathrm{keV}$ PC mode small FOV of all the orbits for all the observations. (A color version of this figure is available in the online journal.)

6. The combined WT and PC $0.3-10 \mathrm{keV}$ binned light curves in units of count rate with the best model obtained during the light curve analysis (Figure 9). The dashed black lines indicate the position at the time of the light curve breaks. Sometimes the fitted values for the time breaks may not exactly match the spectral intervals fitted on the spectra page. This is because the time boundaries used to derive the spectra correspond to the nearest light curve bin boundary. In this Xspec-style plot, the bottom part reproduces the residual of the data versus the model.

7. The combined WT and PC $0.3-10 \mathrm{keV}$ spectrum of each interval, defined during the light curve analysis (Figure 10). These spectra refer to the continuum emission of the source, as explained in Section 5.2.

\subsubsection{FITS}

The input FITS files for the light curve and spectral analyses and the summary of the results of both analyses can be downloaded. The user will find the following:
1. The fully corrected and background-subtracted PC and WT binned light curves, in the entire energy range of $0.3-10 \mathrm{keV}$ and in the two sub-ranges of $0.3-2$ and $2-10 \mathrm{keV}$. These curves are both in units of count rate and $\mathrm{erg} \mathrm{cm}^{-2} \mathrm{~s}^{-1}$. Together with them, the source PC and WT hardness ratio is also present. All these curves have been used to generate the images previously described.

2. The fully corrected PC and WT unbinned light curves of the source and the background in the entire energy range $0.3-10 \mathrm{keV}$ and in the two sub-ranges $0.3-2$ and $2-10 \mathrm{keV}$;

3. PHA spectra for the source and the background and the corresponding response matrix for one or more time intervals and for both the WT and PC modes. The time intervals were determined with the light curve analysis, as described in Section 5.2.

4. The information file: XRTINFO, XRTFLDREG, and XRTFITINFO extensions. XRTINFO contains the name of the files used for the data reduction and analysis, information used to create the final light curve for each of 


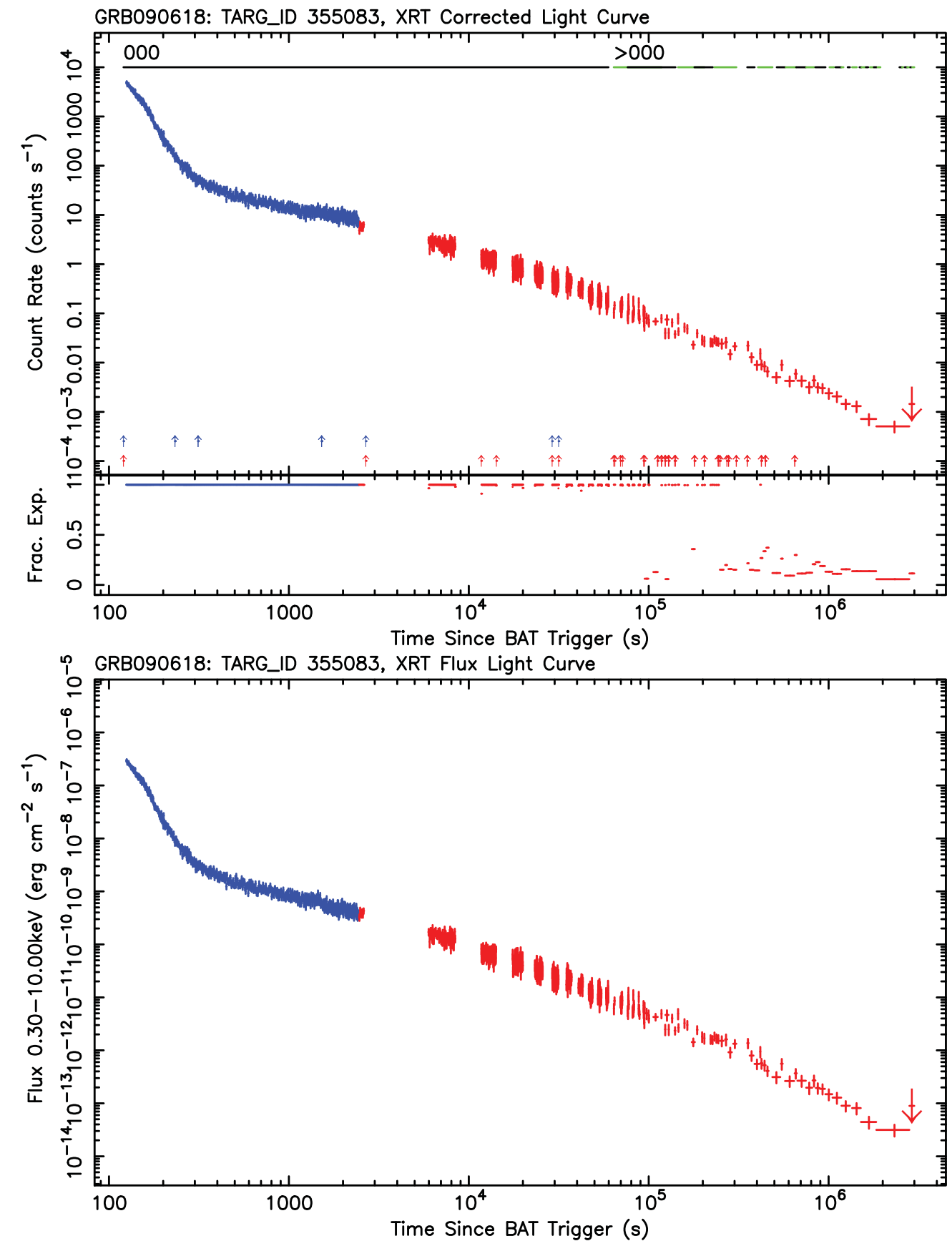

Figure 7. Combined PC (blue) and WT (red) mode binned light curve in unit of count rate (top panel) and of $\mathrm{erg} \mathrm{cm}^{-2} \mathrm{~s}^{-1}$ (bottom panel) in the $0.3-10 \mathrm{keV}$ energy range. The fractional exposure shown below the count rate light curve is defined as the exposure time divided by the bin duration.

(A color version of this figure is available in the online journal.)

the orbits, such as the start and stop times, the source and background extracted counts (in the $0.3-10 \mathrm{keV}$, $0.3-2 \mathrm{keV}$, and $2-10 \mathrm{keV}$ ranges), the spatial and temporal correction factors, the count rate to flux conversion factor, and details of the source and background extraction regions. XRTFLDREG collects all the data for the detected FOV sources found in the PC event file by Ximage: position, intensity, and probability of detection, as well as the size of the region associated with each source. This information is necessary to create the series of exclusion regions that are used to extract the source and background light curves and spectra (see Section 5.2). XRTFITINFO contains the results of both the light curve and spectral analyses. For every fit, it reports the times of the interval that has been fitted, the mode (WT or PC) used to collect the data, the fit statistics (degrees of freedom and $\chi^{2}$ value), which model has been adopted (in Xspec style), and who performed the fit (in automatic by the pipeline or by the intervening user if the automatic fit failed or was not optimal). Values up to six parameters are reported. These parameters refer to different quantities, based on the type of fit, e.g., light curve or spectral fit: (1) first slope or Galactic absorption, (2) first break or intrinsic absorption, (3) second slope or spectral index, (4) second break or spectral model normalization, (5) third slope, (6) light curve model normalization. For the 


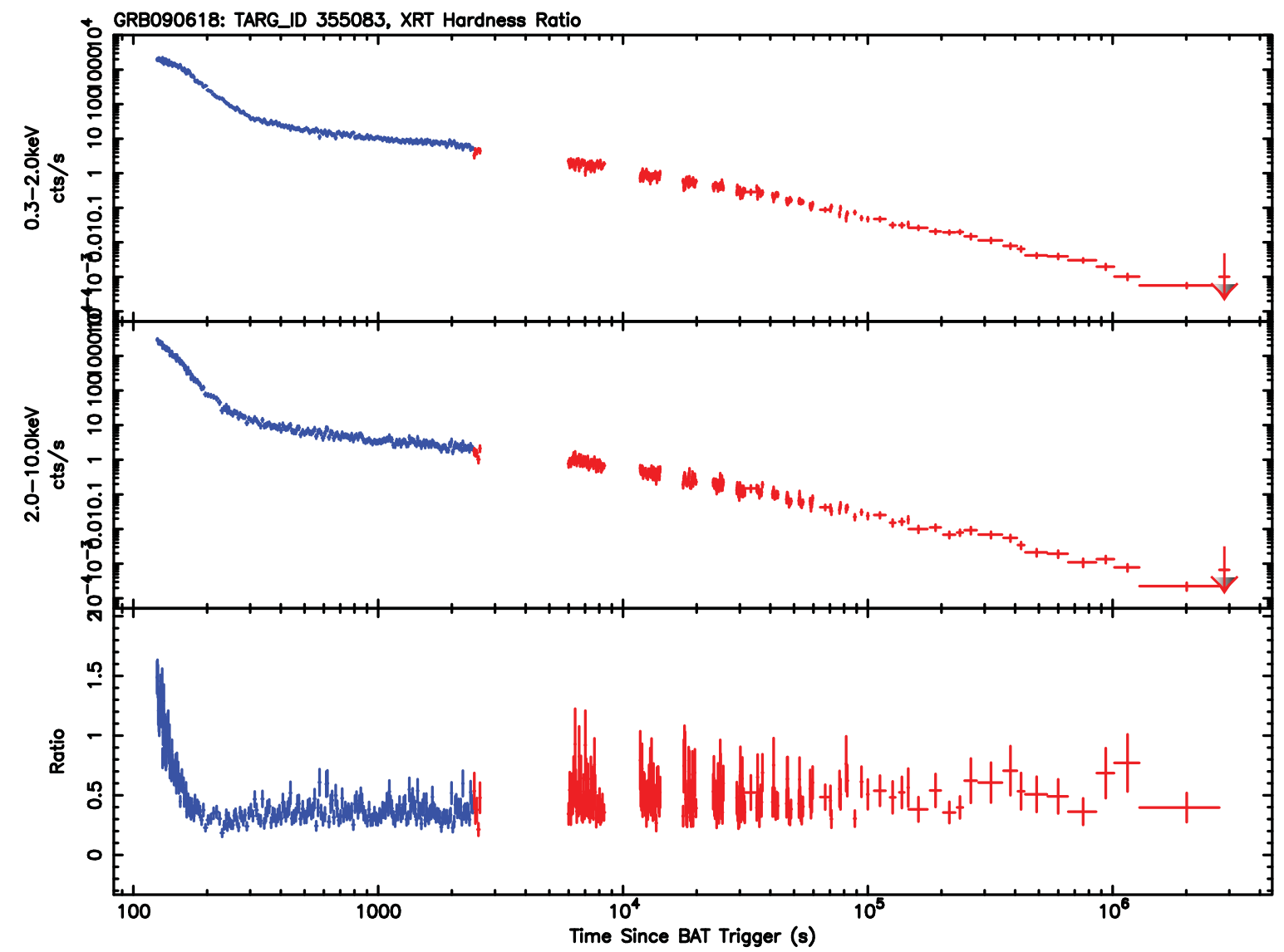

Figure 8. Hardness ratio for the PC (blue) and WT (red) data. The hardness ratio, defined as $\mathrm{H} / \mathrm{S}$, where $\mathrm{S}$ and $\mathrm{H}$ are the number of counts in the soft $(0.3-2 \mathrm{keV})$ and hard $(2-10 \mathrm{keV})$ bands, respectively. The count rate light curves in the two bands are plotted in the top and central panels, while the hardness ratio is plotted in the bottom panel. We adopted the binning of the hard band light curve to group the soft band light curve.

(A color version of this figure is available in the online journal.)

spectral analysis, the value of the observed flux is given. All these quantities come with the upper and lower value, determined after adding and subtracting the related $1 \sigma$ error, respectively.

\subsection{UVOT}

Since no spectral analysis is performed on the UVOT data, only two sets of gif images are generated by uvotgrblc, providing spatial and temporal information. The sets include the following:

\subsubsection{Images}

1. The summed image with the highest signal-to-noise ratio centered on the source and with an FOV of $\sim 4.5$. For this particular OBS_ID and filter, the aperture used for photometry, the background region, and the excluded field sources are indicated with a solid blue circle, a blue dashed region, and red dashed circles, respectively (Figure 11, left panel).

2. The earliest exposure centered on the source and with an FOV of $\sim 7: 5$. Overplotted on this image are the BAT and XRT error circles (Figure 11, right panel). For GRBs, sometimes this exposure is obtained when the satellite is still settling. This will result in sources with elongated trails, as in the case presented in Figure 11.

3. The light curves in units of magnitude and $\mathrm{erg} \mathrm{cm}^{-2} \mathrm{~s}^{-1}$ (Figure 12). For each filter, the conversion factors from flux density to integrated flux have been estimated as explained in Section 6. In the plots, down arrows indicate the $3 \sigma$ upper limit for that bin. The colors in the light curve are in units of erg $\mathrm{cm}^{-2} \mathrm{~s}^{-1}$ to match those used in the magnitude light curve: red $=\mathrm{V}$, green $=\mathrm{B}$, blue $=\mathrm{U}$, light blue $=$ $\mathrm{W} 1$, magenta $=\mathrm{M} 2$, yellow $=\mathrm{W} 2$, orange $=$ WHITE. We want to point out that the light curves may contain the first exposure in the $V$ filter, which can be affected by the trailing issue (see the previous bullet). This will reduce the real count rate and, consequently, will affect the estimate of the magnitude and the flux. We urge the user to discard this point in the light curves if the trailing effect is seen in the image, as in Figure 11, and to regenerate the light curves with a customized analysis.

\subsubsection{FITS}

The only UVOT FITS file available for download is the input file used to generate the two light curve plots (see above). For each exposure or sum of exposures it reports the final photometric value for the detection or the upper limit. It is produced by uvotsource, but is modified by uvotgrblc to contain additional information, such as the integrated flux (with error), the estimated sigma detection, the inner and outer radii of the background region, the aperture used to extract the source photometry, the aperture correction factor, and the basic information about the star used to determine this factor. As in the case of the light curve plots, the FITS file may also contain 


\section{GRB090618: XRT PC/WT Automatic Light Curve Fit Double Broken Power-Law}

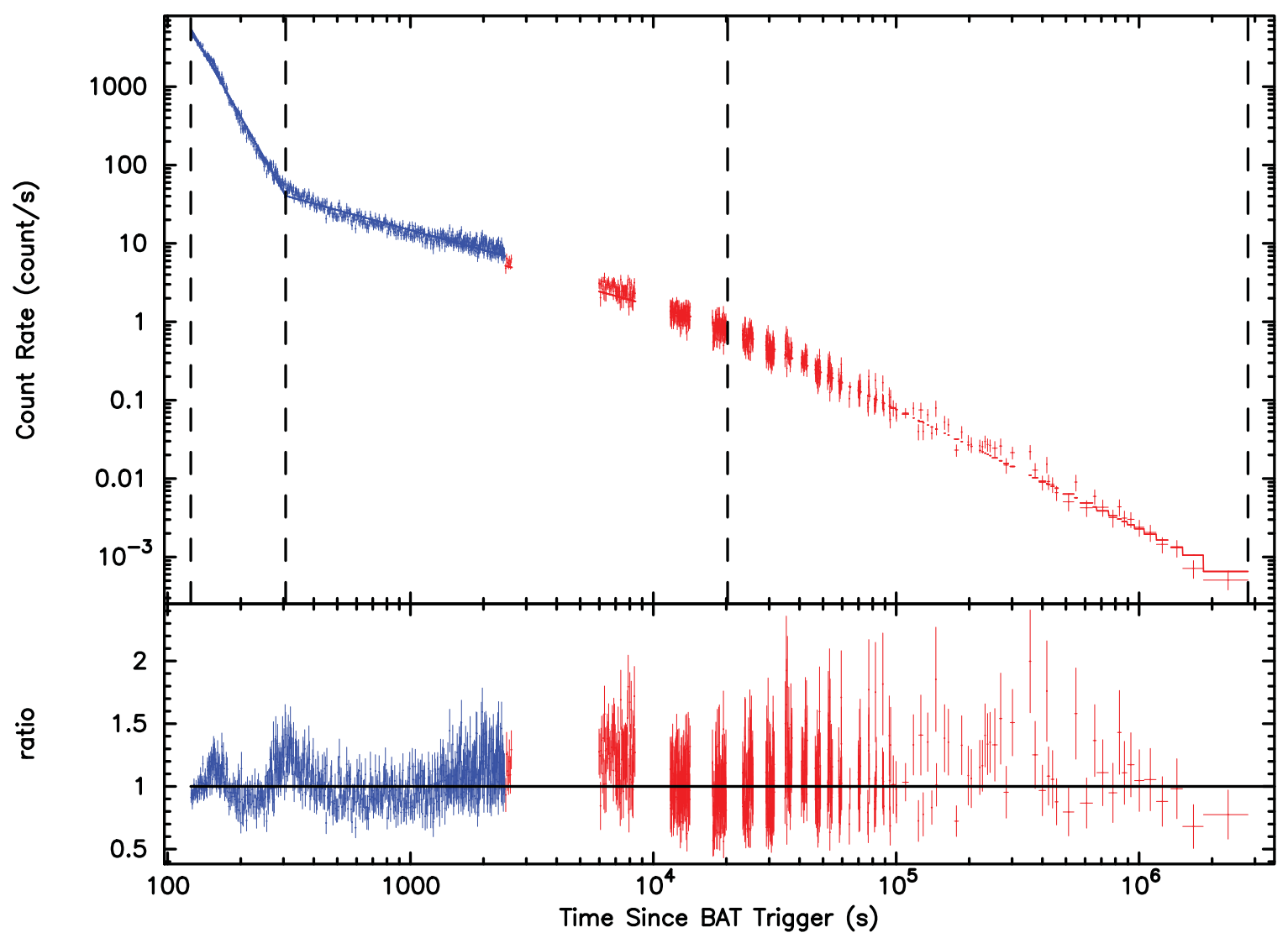

Figure 9. XRT light curve analysis. The dashed lines indicate the position of the breaks found by the script xrtgrblcspec, using the double broken power law model within Xspec. The lower panel shows the ratio obtained by dividing the data by the folded model to highlight deviations.

(A color version of this figure is available in the online journal.)

the value from the first exposure in the $V$ filter. This should be ignored if Swift was still settling at the time of the data collection the user must perform a customized analysis to obtain reliable results.

\subsection{Combined XRT and BAT$$
\text { 6.4.1. Images }
$$

The processing script swifthlprod also generates two plots that show the combined XRT and BAT light curves in the 0.3-10 and 2-10 keV energy bands (Figure 13). The Bayesian block BAT light curve (in black) is combined with the WT (in blue) and PC mode (in red) binned light curve. The BAT light curve conversion to these two energy bands is explained in Section 5.1.

\section{CATALOG: PIPELINE AND ADDITIONAL INFORMATION RETRIEVAL}

Once the GRB analysis is completed, some quantities related to the XRT and UVOT analysis are extracted from the files generated by the pipeline and imported in the Swift Gamma Ray Burst Catalog (see Section 2).

For the XRT, this table provides information regarding the following.

1. The first 100 count interval: the corrected $0.3-10 \mathrm{keV}$ count rate, the start and stop time, exposure and mode used (PC, WT, or both).
2. The first $300 \mathrm{~s}$ interval: the corrected count rates in the total $0.3-10 \mathrm{keV}$ band, as well as the three sub-bands (0.3-1 keV, 1-2 keV, and 2-10 keV), the start and stop time, exposure, mode used, and the hardness ratios between the sub-bands. The rate is estimated as follows: (a) if there are at least 100 counts detected across $300 \mathrm{~s}$, then the time range corresponds to the arrival of these counts, (b) if there are less than 100 counts, then the time range is the full observation (if there is not a detection, an upper limit is provided), (c) if the observation starts $400 \mathrm{~s}$ or more after the GRB trigger time, then no value is reported.

In addition, the table provides the presence of flares and light curve changes in the continuum and the most accurate position observed with the XRT. The latter is derived from the UK Swift Science Data Centre Web site at the University of Leicester (http://www.swift.ac.uk/xrt_positions/). The position is typically enhanced by correcting the astrometric reference of the XRT. This will reduce the absolute systematic error on the position to 1". 4 . The correction is obtained by "matching the stars seen by UVOT with the USNO-B1 catalog and using the known mapping between the UVOT detector and the XRT detector." " A detailed description of the method used can be found in Goad et al. (2007) and Evans et al. (2009).

\footnotetext{
7 Swift Science Data Centre Website, http://www.swift.ac.uk/xrt_positions/ docs.php.
} 

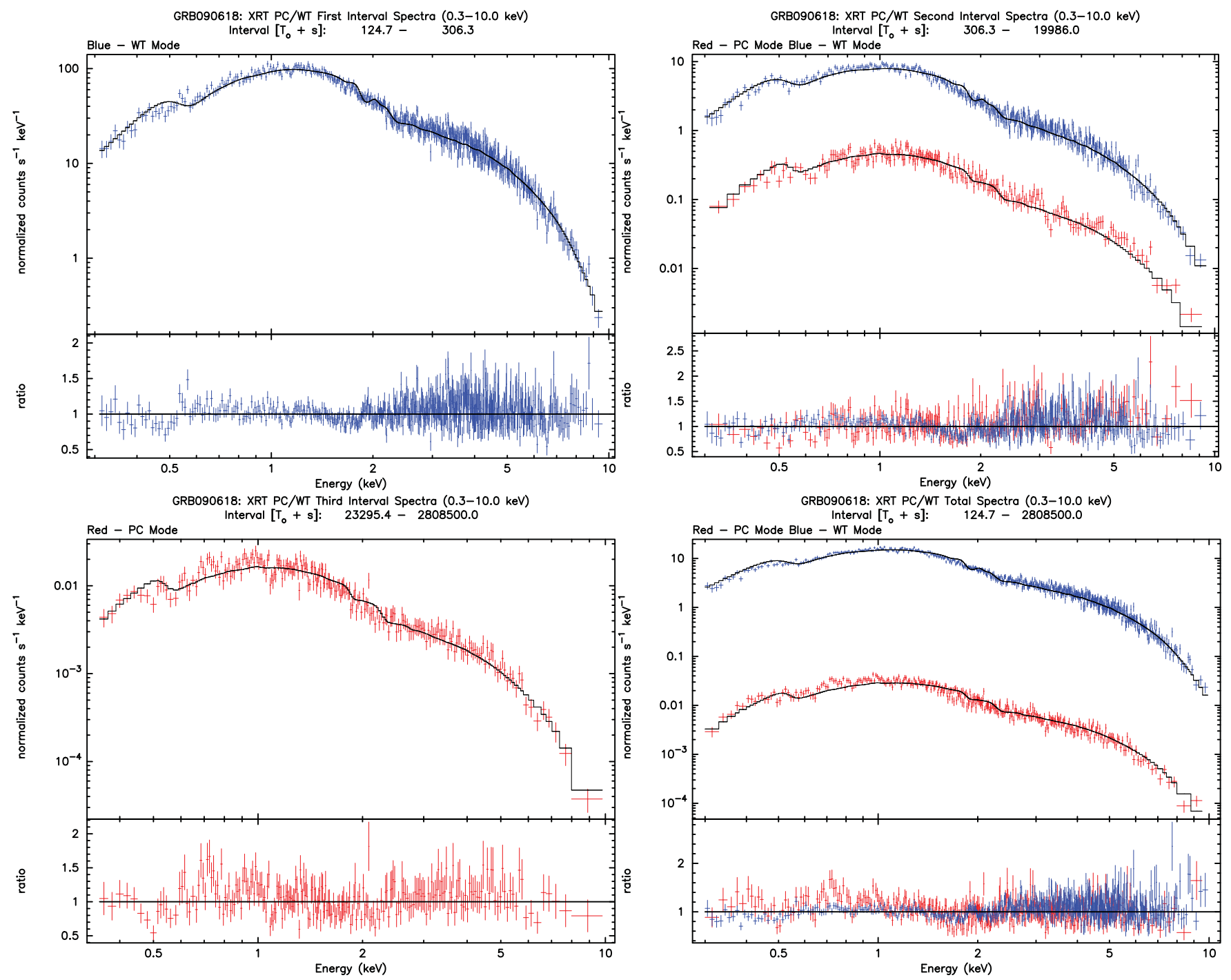

Figure 10. Plots of the XRT spectral analysis at different time intervals. The time breaks were obtained by fitting the light curve with a double broken power law model in Xspec (see Figure 10). As in previous figures, the blue and red points refer to WT and PC mode data, respectively.

(A color version of this figure is available in the online journal.)

For the UVOT, our Swift Gamma Ray Burst Catalog reports: (1) the observed magnitude and monochromatic flux of the first observation in each filter; the start and stop time and exposure for these observations are also given and (2) the best position derived from the GCN circulars.

The Catalog contains a substantial amount of information related to the BAT instrument: (1) position, (2) significance of the detection, (3) type of trigger (image or rate), (4) length of the T90 and T50, (5) the start and stop of the T100, (6) T100 fluence in units of erg $\mathrm{cm}^{-2}$ in the full energy range of $15-150 \mathrm{keV}$ as well as four sub-ranges $(15-25,25-50,50-100,100-150 \mathrm{keV})$ and the spectral model used to estimate those quantities (power law or exponential cutoff power law), (7) $1 \mathrm{~s}$ peak flux in units of erg $\mathrm{cm}^{-2} \mathrm{~s}^{-1}$ and of photons $\mathrm{cm}^{-2} \mathrm{~s}^{-1}$ both in the full energy range of $15-150 \mathrm{keV}$ and in the four sub-ranges, the time of the peak and the spectral model, (8) results of the spectral analysis of the T100 interval in the $15-150 \mathrm{keV}$ band using the power law or the exponential cutoff power law model (spectral slope, energy of the cutoff, reduced $\chi^{2}$, and degrees of freedom), and (9) hardness ratios of the fluence in the $25-50 \mathrm{keV}$ and $50-100 \mathrm{keV}$ ranges with respect to the fluence in the $15-25 \mathrm{keV}$.
Those quantities are regularly published in the preliminary reports from the BAT team ground-based analysis (http://gcn.gsfc.nasa.gov/swift_gnd_ana.html). The Swift GRB Catalog simply collects them for an easier comparison and usage. The BAT team, however, has run a more detailed and GRBspecific analysis. This work led to the publication of two catalogs (the First and the Second Swift BAT Gamma Ray Burst Catalogs, Sakamoto et al. 2008 and Sakamoto et al. 2011, respectively). The Swift GRB Catalog has been updated with the results from these peer reviewed analyses, in order to have more reliable and precise information.

The Swift GRB Catalog also contains the following information from the GCN circulars or the literature: (1) the redshift (value, error, and type, i.e., if from absorption and/or from emission lines, or from photometric measurements), (2) the detection using ground-based telescopes (radio, infrared, and optical), (3) the trigger or follow-up observations with other $\mathrm{X}$-ray and $\gamma$-ray satellites, (4) the presence of a supernova signature, (5) the association of the afterglow with a host galaxy (position, type of galaxy, redshift, and distance from the afterglow). 
GRB090618: UVOT, TARG_ID $=355083$, Highest $S / \mathrm{N}$ Image OBS_ID $=00355083000$, FILTER=WHITE

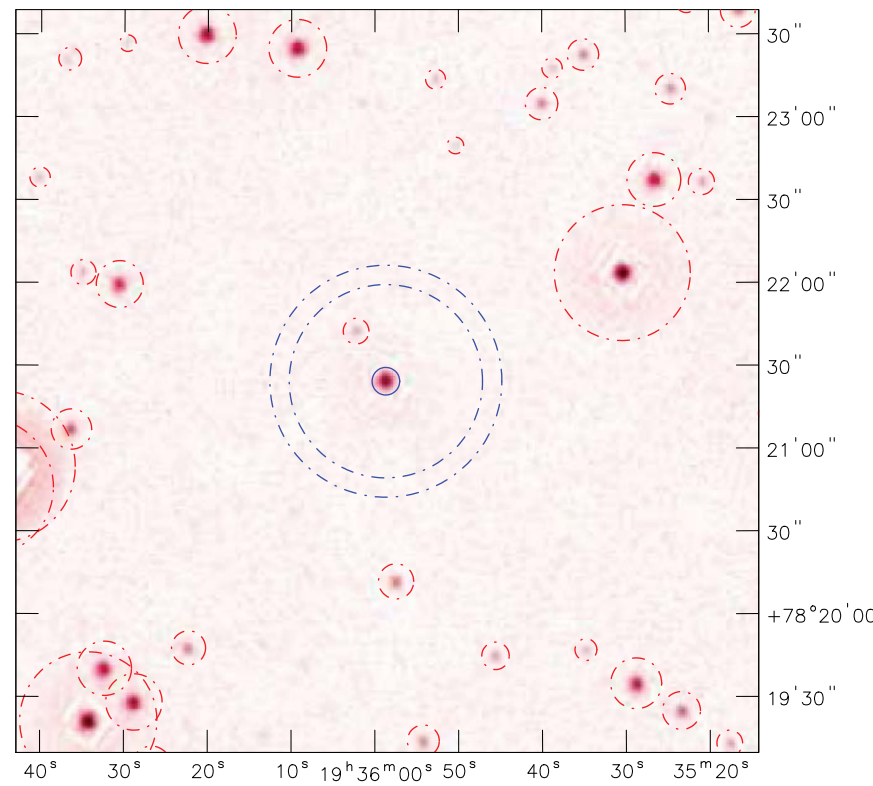

GRB090618: UVOT, TARG_ID $=355083$, Finding Chart Image OBS_ID $=00355083000$, FILTER=V, EXPID $=267006625$

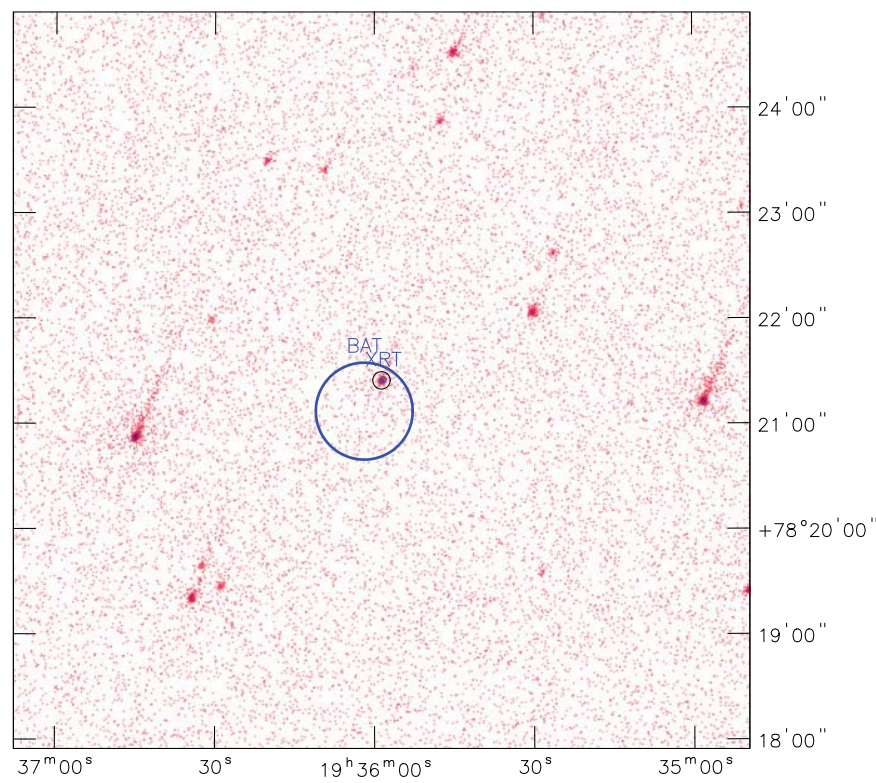

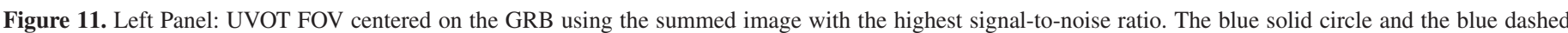

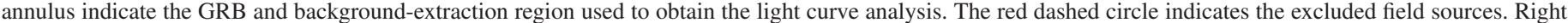

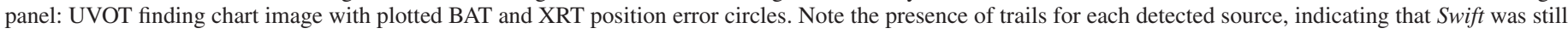
settling when those data were recorded.

(A color version of this figure is available in the online journal.)
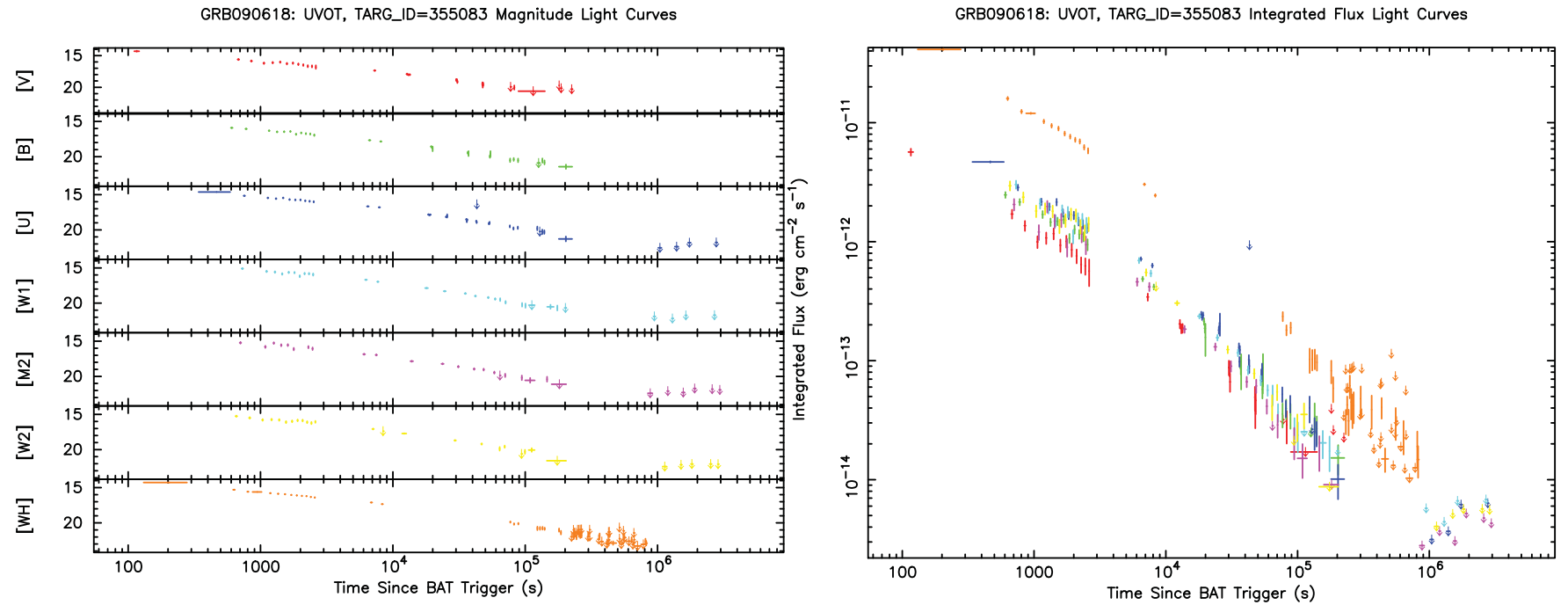

Figure 12. UVOT light curve in units of magnitude (left) and $\mathrm{erg} \mathrm{cm}^{-2} \mathrm{~s}^{-1}$ (right). Each color corresponds to a different filter: red $=\mathrm{V}$, green $=\mathrm{B}$, blue $=\mathrm{U}$, light blue $=\mathrm{W} 1$, magenta $=\mathrm{M} 2$, yellow $=\mathrm{W} 2$, orange $=$ WHITE. As mentioned in the text, the user should ignore the first data point in the $V$ filter if the satellite was still settling during the exposure (see also the previous figure).

(A color version of this figure is available in the online journal.)

\section{COMPARISON OF PIPELINE RESULTS WITH PUBLISHED CATALOGS}

In order to test the reliability of the pipeline, a comparison with previously published results is mandatory. In particular, we focused our attention on works where the aim was to produce a catalog for one of the instruments on board Swift, i.e., Sakamoto et al. (2008) for the BAT catalog, Evans et al. (2009) for the XRT catalog, and Roming et al. (2009) for the UVOT GRB catalog. A consistency check was performed on a sample of 27 GRBs that encompasses a wide range of behaviors in each instrument. For all the instruments we selected both long and short GRBs, as well as very bright and barely detected ones, and those also observed in the earliest and in the latest part of Swift's mission. We tested the pipeline for some "extreme" cases: for the BAT, a few GRBs have a detected precursor while others were observed with a small partial coding fraction; for the XRT, we selected observations with high flickering between the WT and PC modes, with strong pile-up in both modes, and with the dead columns crossing the PSF on the side or perfectly on center; for the UVOT, we analyzed the emission from GRBs in crowded areas. 

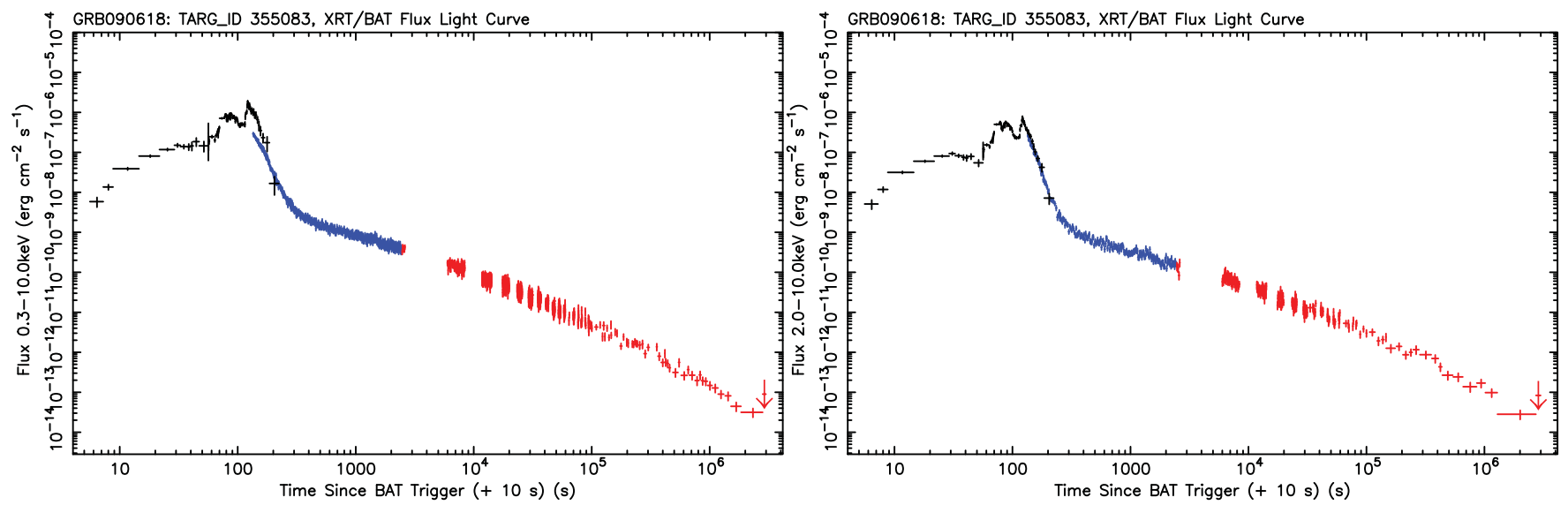

Figure 13. Combined BAT (black) and XRT (WT mode: blue and PC mode: red) light curves in units of erg $\mathrm{cm}^{-2} \mathrm{~s}^{-1}$. The BAT light curve is converted into the $0.3-10 \mathrm{keV}$ range (left panel) and the $2-10 \mathrm{keV}$ range (right panel).

(A color version of this figure is available in the online journal.)

Since the BAT and UVOT published catalogs extend up to 2007 June 16, a comparison with the most recent observations was only performed for the XRT data.

We chose as good testing parameters the fluence in the $15-25,25-50,50-100,100-150 \mathrm{keV}$ ranges for the BAT, the optical/UV magnitudes for the UVOT, and the light curve slope decay for the XRT.

Notes on the selection criteria that were used are as follows:

1. To estimate the BAT fluence, we considered the flux in each energy range obtained by fitting the spectrum in Xspec and we divided it by the exposure time.

2. While Roming et al. (2009) performed the photometry on the individual sky images, regardless if a detection or an upper limit was found, our pipeline combines the individual sky images if consecutive upper limits are obtained in order to lower the detection/upper limit threshold. For this reason, only the optical/UV magnitudes in the individual sky images that have a detection in both Roming's and our pipeline are considered.

3. For the XRT comparison we used an approach similar to the one described in Evans et al. (2007). We downloaded the count rate light curve for all the GRBs from the online repository that was created by the University of Leicester. We performed the light curve analysis, finding the values of the time breaks where the light curve shows a change with respect to a constant (power law) decay. For each phase of constant decay, we estimated the slope of the power law decay. The same quantity was calculated in the analysis of the light curves produced by our pipeline, but with the assumption that the position of the time breaks is the same as the one previously estimated. In this way, a difference in the slope decay does not depend on the position of the time break.

For all the testing parameters we evaluated the offset (in $\sigma$ ) between the values obtained by our pipeline and from the other public catalogs. The offset is defined as $\left(\mathrm{val}_{\text {pipe }}-\mathrm{val}_{\mathrm{cat}}\right) / \sigma_{\text {tot }}$, where $\sigma_{\text {tot }}$ is the propagated standard deviation and is defined as $\left(\sigma_{\text {pipe }}^{2}+\sigma_{\text {cat }}^{2}\right)^{-1 / 2}$. The offset distributions for the BAT fluence, the UVOT optical/UV magnitude, and the XRT light curve slope are shown in Figure 14.

The plots show the reliability of our pipeline: the distribution of the $\sigma$ for the three quantities are Gaussian-like, with the peak close to zero. The standard deviations are $0.14,0.35$, and 0.53 for the BAT, UVOT, and XRT distributions, respectively, meaning that $95 \%$ of the values produced by our pipeline are within $0.27 \sigma, 0.69 \sigma$, and $1.04 \sigma$ from the values reported in the public catalogs.

The discrepancies between the Swift GRB Catalog and other published works can be due to a large variety of factors. We name the most relevant as follows:

1. Calibration databases and software version releases (all),

2. Data reduction filtering (all),

3. Source and background-extraction region sizes (XRT and UVOT),

4. Count rate correction methods (XRT and UVOT), and

5. Background position (UVOT).

\section{SUMMARY AND CONCLUSIONS}

We have developed a semi-automatic pipeline that performs the data reduction and analysis of the three instruments on board Swift. The pipeline has been used to generate a uniform and complete analysis of all the GRBs observed by Swift and it will continue to be used as long as the mission is in operation. For the very recent GRBs, and for the ones that will be discovered or observed in the near future, the pipeline has used and will use the updated release of the CALDB files. The software tools that swifthlprod calls are also updated when bugs are fixed or new improvements are included. This will potentially affect the uniformity of the output products. For these reasons, we are planning to regenerate those products when major changes occur in the CALDB files or as released in the software tools.

As mentioned in Section 1, there are many places where the user can retrieve data products for the GRBs observed by Swift. While our database is very much in sync with the products available at the University of Leicester Web site for the XRT data analysis, additional files are available for the BAT, especially regarding the spectral analysis: the PHA fits files during each single phase (pre-slew, slew, and post-slew, if applicable) can be downloaded, together with the respective response files, so that the user can perform a customized analysis. The main difference regarding the UVOT data products with respect to what the user can find in the Swift Burst Ground-Analysis Web site or at the "First Swift UVOT GRB Afterglow catalog" is that our light curves cover the full list of GRBs observed by 

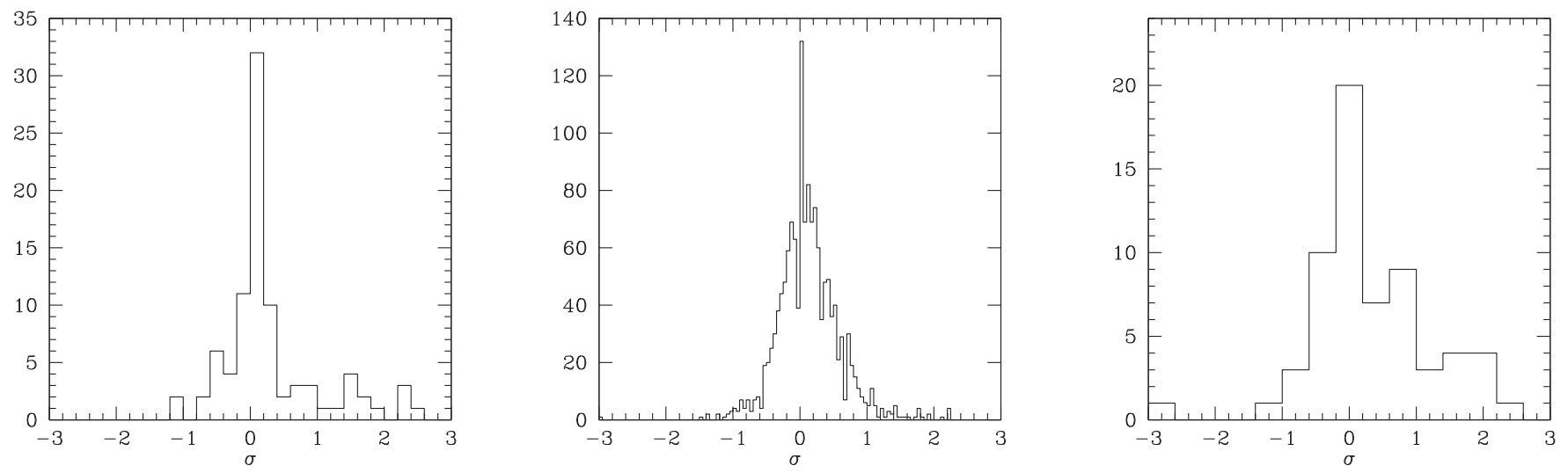

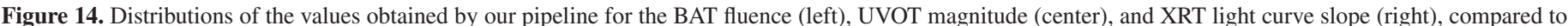
the values reported in public catalogs.

Swift and have been obtained by: (1) carefully selecting specific background and source extraction regions, (2) using field stars in the estimate of the aperture-correction, and (3) regenerating the level 2 products using updated CALDB files.

The results of the pipeline run were combined with data from the GCN circulars, the literature, and the BAT ground-based analysis to populate the Swift GRB Catalog, whose purpose is to generate a "summary" of the characteristics of those GRBs. We believe that this catalog is a useful tool for the public since it is constantly updated (typically within 1-2 business days after a GRB has been triggered or observed) and it collects information not specifically related to the Swift data analysis. As mentioned in Section 7, the catalog provides information regarding the measured redshift, the presence of a host galaxy, and if the GRB was observed in other wavelengths (radio, infrared, or optical) and by other high energy missions. This can help the user in selecting only those GRBs that meet specific criteria.

Another important aspect that was taken into account in developing the pipeline, is its versatility: indeed, it can be of help to perform an analysis of very different types of pointlike sources, such as AGNs, pulsars, etc. This is particularly true when the user wants to analyze XRT and UVOT data (for an analysis of the BAT data for sources that are not GRBs, we suggest using the batsurvey tool developed by the BAT team). Changing the parameter settings for either swifthlprod, xrtgrblc, and/or uvotgrblc, one can easily optimize the use of those scripts to match specific needs.

The script that handles the data reduction and analysis (swifthlprod), the results for each of the observed GRB (database), and the Swift GRB catalog are available for downloading and querying at the HEASARC Web site, while the single scripts that perform the XRT and UVOT analysis (xrtgrblc and uvotgrblc) are already part of the HEAsoft release.

\section{REFERENCES}

Barthelmy, S. D., Barbier, L. M., Cummings, J. R., et al. 2005, Space Sci. Rev., 120,143

Breeveld, A. A., Curran, P. A., Hoversten, E. A., et al. 2010, MNRAS, 406, 1687

Burrows, D. N., Hill, J. E., Nousek, J. A., et al. 2005, Space Sci. Rev., 120, 165

Evans, P. A., Beardmore, A. P., Page, K. L., et al. 2007, A\&A, 469, 379

Evans, P. A., Beardmore, A. P., Page, K. L., et al. 2009, MNRAS, 397, 1177

Evans, P. A., Willingale, R., Osborne, J. P., et al. 2010, A\&A, 519A, 102

Gehrels, N., Chincarini, G., Giommi, P., et al. 2004, ApJ, 611, 1005

Goad, M. R., Tyler, L. G., Beardmore, A. P., et al. 2007, A\&A, 476, 140

Hill, J. E., Burrows, D. N., Nousek, J. A., et al. 2004, SPIE, 5165, 217

Klebesadel, R. W., Strong, I. B., \& Olson, R. A. 1973, ApJ, 182, L85

Meegan, C. A., Fishman, G. J., Wilson, R. B., et al. 1992, Nature, 355, 143

Moretti, A., Campana, S., Mineo, T., et al. 2005, SPIE, 5898, 348

Poole, T. S., Breeveeld, A. A., Page, M. J., et al. 2008, MNRAS, 383, 627

Puccetti, S., Capalbi, M., Giommi, P., et al. 2011, A\&A, 528A, 122

Romano, P., Campana, S., Chincarini, G., et al. 2006, A\&A, 456, 917

Roming, P. W. A., Kennedy, T. E., Mason, K. O., et al. 2005, Space Sci. Rev., 120,95

Roming, P. W. A., Koch, T. S., Oates, S. R., et al. 2009, ApJ, 690, 163

Sahu, K. C., Livio, M., Petro, L., et al. 1997, Nature, 387, 476

Sakamoto, T., Barthelmy, S. D., Barbier, L., et al. 2008, ApJS, 195, 2

Sakamoto, T., Barthelmy, S. D., Baumgartner, W. H., et al. 2011, ApJS, 175,179

Sakamoto, T., Hill, J. E., Yamazaki, R., et al. 2007, ApJ, 669, 1115

Wijers, R. A. M. J., Rees, M. J., \& Meszaros, P. 1997, MNRAS, 288, L51 